\title{
A new picture fuzzy divergence measure based on Jensen-Tsallis information measure and its application to multicriteria decision making
}

\author{
Ratika Kadian $^{1} \cdot$ Satish Kumar ${ }^{1}$
}

Received: 18 November 2020 / Accepted: 6 January 2021 / Published online: 14 March 2021

(c) The Author(s), under exclusive licence to Springer Nature Switzerland AG 2021

\begin{abstract}
Picture Fuzzy Sets (PFSs) originated by Cuong and Kreinovich are more capable to capture uncertain, inconsistent and vague information in multi-criteria decision making. In this paper, we propose a new picture fuzzy divergence measure based on Jensen-Tsallis function between PFSs. Further, the concept has been extended from fuzzy sets to novel picture fuzzy divergence measure. Besides the validation of the proposed measure, some of its key properties with specific cases are additionally talked about. The performance of the proposed measure is compared with other existing measures in the literature. Some illustrative examples are provided in the context of novel rapacious COVID-19 and pattern recognition which demonstrate the adequacy and practicality of the proposed approach in solving real-life problems.
\end{abstract}

Keywords Picture fuzzy set · Jensen inequality $\cdot$ Coronavirus disease $\cdot$ MCDM $\cdot$ COVID-19

\section{Introduction}

Zadeh (1965) introduced the idea of the fuzzy set to quantify the vague and uncertain information and applied them in decision making problems from different angles (Chen et al. 2012; Chen and Chen 2014; Kadian and Kumar 2020a). Atanassov (1986) introduced a significant generalization of the fuzzy set known as intuitionistic fuzzy set (IFS). Fuzzy set theory is applied in various areas, but in real life, few circumstances happen that cannot be dealt with by a fuzzy set. The idea of the intuitionistic fuzzy set for a component is a membership degree $(\tau)$, non-membership degree $(v)$ and the third component 'hesitancy degree' $(\pi)$ respectively, satisfying $\tau+v+\pi=1$, improved the capability of FSs to handle the uncertain information. The third factor namely 'hesitancy degree' $(\pi)$ was introduced by Atanassov (1999) in the existing

Ratika Kadian

kadian.ratika@mmumullana.org

Satish Kumar

profsatish74@mmumullana.org

1 Department of Mathematics, Maharishi Markandeshwar (Deemed To Be University), Mullana, Ambala 133207, India structure of FSs. Various researchers gave their contribution in this field such as Szmidt and Kacprzyk (2000) developed a similarity measure between IFSs based on the Hamming distance, Xu and Xia (2010) characterized the geometric distance and similarity measures of IFSs for group decision-making problems. Due to their flexibility and successful applications, various researchers have been started research work on Atanassov intuitionistic fuzzy theory. More interesting applications have been developed in various fields, for example, image processing, risk analysis, medical diagnosis (Son and Phong 2016; Srivastava and Maheshwari 2016), decision making (Chen and Chang 2016; Chen et al. 2016; Zeng et al. 2019; Joshi 2020; Kadian and Kumar 2020b).

The transmission of COVID-19 and different mediations additionally had an extraordinary contrary impact on the normal existences of individuals and the solid working of society. Numerous researchers contribute to prevent and control the crisis circumstance of COVID-19. Toğaçar et al. (2020) introduced the deducting technique of COVID-19 using fuzzy color and stacking approaches, Tuite et al. (2020) propose the COVID-19 transmission and relief techniques based numerical model among Canada's population, Sohail and Nutini (2020) presented 
the novel numerical model of determining the time period of COVID-19.

Tackling ambiguous and unsure information in real-life circumstances have consistently been a trouble. A few methodologies have been investigated to address the unpredictability and uncertainty found in real-life measures, for example the theory of fuzzy set (FS). Despite the fact that the IFSs find their applications in various fields, yet at the same time there are numerous circumstances where IFSs can not be applied. Again, this can be better understood by an example on voting where we face such sort of problem: yes, abstain, no and refusal. This limits the area of applications of IFSs. To conquer this circumstance, a new generalized fuzzy set namely picture fuzzy set (PFS) has been proposed by Cuong and Kreinovich (2014). It is a generalization of fuzzy set and intuitionistic fuzzy set with the introduction of the positive $(\tau)$, the negative $(v)$, the neutral $(\pi)$ and the refusal degrees $(\xi=1-\tau-v-\pi)$ demonstrating different possibilities of an element to a given set. The main requirement is that the sum of the degrees must not exceed 1. The picture fuzzy set theory is utilized in many day to day life problems such as voting problems, clustering, decision making, fuzzy inference. Son and Thong (2017) presented a few novel fuzzy clustering algorithm depicting the advantages of utilizing PFSs, Wei (2018) proposed picture fuzzy cross-entropy model for multiple attribute decision-making problems, Ashraf et al. (2019) acquaints various methods to multi-criteria group decision-making problems for picture fuzzy environment.

In this paper, we propose a new picture fuzzy divergence measure based on the generalization of JensenTsallis function. Numerical examples illustrate that the proposed measure is reasonably measuring the degree of dissimilarity between PFSs.

The main objectives and motivation of this paper are:

- A new picture fuzzy divergence measure is proposed based on joint representation of Jensen-Tsallis measure and studied some of its properties.

- A new picture fuzzy divergence measure is introduced to measure the fuzziness degree for PFSs.

- The reliability and flexibility of the proposed measure are proved with the help of examples in the context of COVID-19 and pattern recognition.

- The proposed divergence measure is applied to solve the MCDM problems under the picture fuzzy condition.

The remainder of the paper is designed as follows: Sect. 1 contains the work done by the earlier researchers in the field. In Sect. 2, we introduce a new generalization of Jensen-Tsallis divergence measure and its properties. In Sect. 3, a new picture fuzzy divergence measure based on
Jensen-Tsallis entropy is proposed with basic definitions and few major properties. Along the way, Sect. 4 proposes the numerical examples to demonstrate the applicability and reliability of the proposed technique. Lastly, the conclusion and future scope are drawn in Sect. 5.

\section{Jensen-Tsallis divergence measure}

In the present section, we introduce the Jensen-Tsallis divergence along with their properties for the probabilistic view point.

Let $\triangle_{k}=\left\{E=\left(e_{1}, e_{2}, \ldots, e_{k}\right) ; e_{p} \geq 0, \sum_{p=1}^{k} e_{p}=1\right\}$, $k \geq 2$ be the complete probability distribution set. For some $E \in \triangle_{k}$, Shannon entropy is

$H(E)=-\sum_{p=1}^{k} e_{p} \log e_{p}$

For some $E_{1}, E_{2} \in \Delta_{k}$ with respect to the coefficient weights $\delta_{1}, \delta_{2} \geq 0$ s.t. $\delta_{1}+\delta_{2}=1$, Lin (1991) define the Jensen-Shannon divergence measure as

$\Phi\left(E_{1}, E_{2}\right)=H\left(\delta_{1} E_{1}+\delta_{2} E_{2}\right)-\delta_{1} H\left(E_{1}\right)-\delta_{2} H\left(E_{2}\right)$.

The quantity (2) is non negative and disappear if and only if $E_{1}=E_{2}$ and also a convex function for $E_{1}$ and $E_{2}$. So, it is called Jensen difference arising out of the convex function due to negative of the Shannon entropy.

Further, Tsallis (1988) introduced a generalization of Shannon entropy as

$H_{T}^{\alpha}(E)=\frac{1}{1-\alpha}\left[\left(\sum_{p=1}^{k} e_{p}^{\alpha}\right)-1\right]$

when $\alpha \rightarrow 1$, then Eq. (3) recovers a Shannon entropy and is a concave function of $E$ for $\alpha>0(\neq 1)$.

Now, we introduce a new concept called Jensen-Tsallis divergence based on Eq. (3) and also studied by Kumar and Joshi (2019).

$\Phi\left(E_{1}, E_{2}\right)=\frac{1}{(1-\alpha)} \sum_{p=1}^{k}\left(\left(\delta_{1} e_{p}+\delta_{2} f_{p}\right)^{\alpha}-\delta_{1} e_{p}^{\alpha}-\delta_{2} f_{p}^{\alpha}\right)$

where $\delta_{1}$ and $\delta_{2}$ are the weight coefficients with $\delta_{1}, \delta_{2} \geq 0$ and $\delta_{1}+\delta_{2}=1$.

Particular Cases:

Case 1. If $\alpha \rightarrow 1$, then Eq. (4) becomes

$\Phi\left(E_{1}, E_{2}\right)=\sum_{p=1}^{k}\left(\delta_{1}\left(e_{p}\right) \log \left(e_{p}\right)+\delta_{2}\left(f_{p}\right) \log \left(f_{p}\right)-\left(\delta_{1} e_{p}+\delta_{2} f_{p}\right) \log \left(\delta_{1} e_{p}+\delta_{2} f_{p}\right)\right)$ 
which becomes generalization of Jensen-Shannon J-divergence measure with coefficient weights $\delta_{1}, \delta_{2}$, which is slightly different from Lin (1991).

Case 2. If $\delta_{1}=\delta_{2}=\frac{1}{2}$ in Eq. (5), then

$$
\begin{aligned}
\Phi\left(E_{1}, E_{2}\right)= & \sum_{p=1}^{k}\left(\frac{1}{2}\left(\left(e_{p}\right) \log \left(e_{p}\right)+\left(f_{p}\right) \log \left(f_{p}\right)\right)\right. \\
& \left.-\frac{1}{2}\left(e_{p}+f_{p}\right) \log \left(\frac{e_{p}+f_{p}}{2}\right)\right)
\end{aligned}
$$

which becomes generalization of Jensen-Shannon J-divergence measure.

Case 3. If $\delta_{1}=\delta_{2}=\frac{1}{2}$ in Eq. (4), then

$\Phi\left(E_{1}, E_{2}\right)=\frac{1}{(1-\alpha)} \sum_{p=1}^{k}\left(\left(\frac{e_{p}+f_{p}}{2}\right)^{\alpha}-\frac{1}{2}\left(e_{p}^{\alpha}+f_{p}^{\alpha}\right)\right)$

which is called Jensen-Tsallis J-divergence measure, which is studied by Burbea and Rao (1982).

Definition 2.1 The Hessian matrix of a function $\Gamma\left(e_{p}, f_{p}\right)$ of two variables is defined as

$\operatorname{Hessian}(\Gamma)=\left[\begin{array}{ll}\frac{\partial^{2} \Gamma}{\partial e_{p}^{2}} & \frac{\partial^{2} \Gamma}{\partial e_{p} f_{p}} \\ \frac{\partial^{2} \Gamma}{\partial e_{p} f_{p}} & \frac{\partial^{2} \Gamma}{\partial f_{p}^{2}}\end{array}\right]$

The function $\Gamma$ is said to convex at that point in its domain if $\operatorname{Hessian}(\Gamma)$ is semi positive definite and concave if $\operatorname{Hessian}(\Gamma)$ is semi negative definite at that point. To obtain the maximality of a function $\Gamma$, calculate the first order derivative partially and equate to zero to obtain critical points.

Properties of Jensen-Tsallis divergence: The defined distance measure Eq. (4) between $E_{1}$ and $E_{2}$ in $\Delta_{k}$ satisfies the following properties and these properties are proposed by Kullback and Leibler (1951):

1. $\Phi\left(E_{1}, E_{2}\right) \geq 0$ with the equality $E_{1}=E_{2}$;

2. $\Phi\left(E_{1}, E_{2}\right)$ is a convex function of $E_{1}$ and $E_{2}$.

Proof 1 . First prove that $\Phi\left(E_{1}, E_{2}\right) \geq 0$. Since, the function defined by (3) is a concave function, therefore, for any $E_{1}$ and $E_{2}$ in $\triangle_{k}$, Jensen inequality implies

$H_{T}^{\alpha}\left(\delta_{1} E_{1}+\delta_{2} E_{2}\right) \geq \delta_{1} H_{T}^{\alpha}\left(E_{1}\right)+\delta_{2} H_{T}^{\alpha}\left(E_{2}\right)$.

$$
\begin{aligned}
& \frac{1}{1-\alpha}\left[\sum_{p=1}^{k}\left(\delta_{1} e_{p}+\delta_{2} f_{p}\right)^{\alpha}-1\right] \\
& \geq \delta_{1} \frac{1}{1-\alpha}\left[\sum_{p=1}^{k}\left(e_{p}\right)^{\alpha}-1\right]+\delta_{2} \frac{1}{1-\alpha}\left[\sum_{p=1}^{k}\left(f_{p}\right)^{\alpha}-1\right] \\
& \Rightarrow\left[\sum_{p=1}^{k}\left(\delta_{1} e_{p}+\delta_{2} f_{p}\right)^{\alpha}-1\right] \geq\left[\sum_{p=1}^{k} \delta_{1}\left(e_{p}\right)^{\alpha}-\delta_{1}\right]+\left[\sum_{p=1}^{k} \delta_{2}\left(f_{p}\right)^{\alpha}-\delta_{2}\right] \\
& \Rightarrow\left[\sum_{p=1}^{k}\left(\delta_{1} e_{p}+\delta_{2} f_{p}\right)^{\alpha}-1\right] \geq\left[\sum_{p=1}^{k} \delta_{1}\left(e_{p}\right)^{\alpha}+\sum_{p=1}^{k} \delta_{2}\left(f_{p}\right)^{\alpha}-\delta_{1}-\delta_{2}\right] \\
& \Rightarrow\left[\sum_{p=1}^{k}\left(\delta_{1} e_{p}+\delta_{2} f_{p}\right)^{\alpha}-1\right] \geq\left[\delta_{1} \sum_{p=1}^{k}\left(e_{p}\right)^{\alpha}+\delta_{2} \sum_{p=1}^{k}\left(f_{p}\right)^{\alpha}-\left(\delta_{1}+\delta_{2}\right)\right]
\end{aligned}
$$

Using $\delta_{1}+\delta_{2}=1$ in Eq. (12), then

$$
\Rightarrow\left[\sum_{p=1}^{k}\left(\delta_{1} e_{p}+\delta_{2} f_{p}\right)^{\alpha}-1\right] \geq\left[\delta_{1} \sum_{p=1}^{k}\left(e_{p}\right)^{\alpha}+\delta_{2} \sum_{p=1}^{k}\left(f_{p}\right)^{\alpha}-1\right]
$$

This implies

$\sum_{p=1}^{k}\left(\delta_{1} e_{p}+\delta_{2} f_{p}\right)^{\alpha} \geq \delta_{1} \sum_{p=1}^{k}\left(e_{p}\right)^{\alpha}+\delta_{2} \sum_{p=1}^{k}\left(f_{p}\right)^{\alpha}$,

$\Rightarrow \Phi\left(E_{1}, E_{2}\right) \geq 0$.

3. Now, we have to show the convexity of function $\Gamma$, where

$\Gamma(e, p)=\left(\delta_{1} e+\delta_{2} f\right)^{\alpha}-\delta_{1} e^{\alpha}-\delta_{2} f^{\alpha}$

Taking derivative partially w.r.t. $e$ and $f$, we get

$$
\begin{aligned}
& \frac{\partial \Gamma}{\partial e}=\alpha\left(\delta_{1} e+\delta_{2} f\right)^{\alpha-1} \delta_{1}-\alpha \delta_{1} e^{\alpha-1} \\
& \frac{\partial \Gamma}{\partial f}=\alpha\left(\delta_{1} e+\delta_{2} f\right)^{\alpha-1} \delta_{2}-\alpha \delta_{2} f^{\alpha-1}
\end{aligned}
$$

Put $\frac{\partial \Gamma}{\partial e}=\frac{\partial \Gamma}{\partial f}=0$ to obtain the critical point. Now, calculating the $\operatorname{Hessian}(\Gamma)$ from definition (2.1) at $e=f$ and utilizing $\delta_{1}+\delta_{2}=1$, we get

$\operatorname{Hessian}(\Gamma)=2 \alpha \delta_{1} \delta_{2}\left[\begin{array}{cc}1 & -1 \\ -1 & 1\end{array}\right]$

which becomes a positive semi definite. This ensures that the function (4) is convex.

\section{Generalized Jensen-Tsallis picture fuzzy divergence measure}

This section provides the definitions and concepts related to PFSs.

Definition 3.1 (Zadeh 1965) A fuzzy set in $X$ (fixed set) is defined by

$$
L=\left\{\left(t, \tau_{L}(t)\right) \mid t \in X\right\}
$$


in which $\tau_{L}: X \rightarrow[0,1]$ is indicated a membership function in $X$ and $\tau_{L}(t) \in[0,1]$.

Definition 3.2 (Atanassov 1986) An intuitionistic fuzzy set $M$ in $X$ (fixed set) is given by

$M=\left\{\left(t, \tau_{M}(t), v_{M}(t)\right) \mid t \in X\right\} ;$

where $\tau_{M}: X \rightarrow[0,1]$ and $v_{M}: X \rightarrow[0,1]$ are indicated the membership and non membership functions in $X$, respectively, and for every $t \in X$ satisfy the condition $0 \leq \tau_{M}(t)+v_{M}(t) \leq 1$. The hesitancy degree for IFS $M$ and $t \in X$ is given by $\pi_{M}(t)=1-\tau_{M}(t)-v_{M}(t)$. Taking $\pi_{M}(t)=0$ implies $v_{M}(t)=1-\tau_{M}(t)$ for all $t \in X$, i.e., IFS $M$ become a fuzzy set.

Definition 3.3 (Atanassov 1986) For each intuitionistic fuzzy set in $X$ (fixed set), if

$\pi_{M}(t)=1-\tau_{M}(t)-v_{M}(t), \forall t \in X$.

Then $\pi_{M}(t)$ is called the hesitancy degree of $t$ to $M$.

Definition 3.4 (Cuong and Kreinovich 2014) A picture fuzzy set in $X$ (fixed set) is given as

$M=\left\{\left(t, \tau_{M}(t), v_{M}(t), \pi_{M}(t)\right) \mid t \in X\right\}$

where $\tau_{M}: X \rightarrow[0,1], v_{M}: X \rightarrow[0,1]$ and $\pi_{M}: X \rightarrow[0,1]$ are indicated the positive, negative and neutral grads in $X$ respectively and for every $t \in X$ satisfy the condition $0 \leq \tau_{M}(t)+v_{M}(t)+\pi_{M}(t) \leq 1$, and the refusal degree for PFS $M$ and $t \in X$ is given by $\xi_{M}(t)=1-\tau_{M}(t)-v_{M}(t)-\pi_{M}(t)$. Taking $\quad \xi_{M}(t)=0$ implies $\pi_{M}(t)=1-\tau_{M}(t)-v_{M}(t)$, i.e., PFS $M$ becomes an intuitionistic fuzzy set. And, again taking $\pi_{M}(t)=0$ implies $v_{M}(t)=1-\tau_{M}(t)$ for all $t \in X$, i.e., IFS $M$ becomes a fuzzy set.

Definition 3.5 (Cuong and Kreinovich 2014) Let $L=$ $\left\{\left(t, \tau_{L}(t), v_{L}(t), \pi_{L}(t)\right)\right\}$ and $M=\left\{\left(t, \tau_{M}(t), v_{M}(t), \pi_{M}(t)\right)\right\}$ be two PFSs, then

1. $L \subseteq M \quad$ if and only if $\forall t \in X, \quad \tau_{L}(t) \leq \tau_{M}(t)$, $v_{L}(t) \geq v_{M}(t)$ and $\pi_{L}(t) \geq \pi_{M}(t)$;

2. $L=M$ if and only if $\forall t \in X, \tau_{L}(t)=\tau_{M}(t)$ and $\tau_{M}(t)=\tau_{L}(t)$

3. $\bar{L}=\left\{\left(\pi_{L}(t), v_{L}(t), \tau_{L}(t)\right) \mid t \in X\right\}$;

4. $L \cup M=\left\{\left(t, \max \left(\tau_{L}(t), \tau_{M}(t)\right), \min \left(v_{L}(t), v_{M}(t)\right)\right.\right.$, $\left.\left.\min \left(\pi_{L}(t), \pi_{M}(t)\right)\right) \mid t \in X\right\}$

5. $L \cap M=\left\{\left(t, \min \left(\tau_{L}(t), \tau_{M}(t)\right), \max \left(v_{L}(t), v_{M}(t)\right)\right.\right.$, $\left.\left.\max \left(\pi_{L}(t), \pi_{M}(t)\right)\right) \mid t \in X\right\}$.

Next, we introduce a new generalized Jensen-Tsallis picture fuzzy divergence measure. First we take $X=\{t\}$ (single element). Then, for some $L, M \in P F S s(X)$, for simplicity, we take $L=\left(\tau_{L}, v_{L}, \pi_{L}, \xi_{L}\right)$ and
$M=\left(\tau_{M}, v_{M}, \pi_{M}, \xi_{M}\right) . \quad$ Since $\quad \tau_{L}+v_{L}+\pi_{L}+\xi_{L}=1$, $0 \leq \tau_{L}, v_{L}, \pi_{L}, \xi_{L}, \tau_{M}, v_{M}, \pi_{M}, \xi_{M} \leq 1, \quad\left(\tau_{L}, v_{L}, \pi_{L}, \xi_{L}\right)$ and $\left(\tau_{M}, v_{M}, \pi_{M}, \xi_{M}\right)$ might be regarded as two probability distributions. We define a dissimilarity measure on $\operatorname{PFSs}(X)$ corresponding to (2) as

$\Phi^{* \alpha}(L, M)=H_{T}^{\alpha}\left(\delta_{1} L+\delta_{2} M\right)-\delta_{1} H_{T}^{\alpha}(L)-\delta_{2} H_{T}^{\alpha}(M)$,

where $H_{T}^{\alpha}(\cdot)$ is Tsallis entropy for $\operatorname{PFS}(\cdot), \alpha>0(\neq 1)$, $\delta_{1}, \delta_{2} \geq 0, \delta_{1}+\delta_{2}=1$ and

$$
\begin{aligned}
& \delta_{1} L+\delta_{2} M \\
& =\left(\delta_{1} \tau_{L}+\delta_{2} \tau_{M}, \delta_{1} v_{L}+\delta_{2} v_{M}, \delta_{1} \pi_{L}+\delta_{2} \pi_{M}, \delta_{1} \xi_{L}+\delta_{2} \xi_{M}\right) .
\end{aligned}
$$

So, the Eq. (15) can be written as

$$
\begin{aligned}
& \Phi^{* \alpha}(L, M)=\frac{1}{(1-\alpha)} \\
& \left(\begin{array}{l}
\left(\delta_{1} \tau_{L}+\delta_{2} \tau_{M}\right)^{\alpha}+\left(\delta_{1} v_{L}+\delta_{2} v_{M}\right)^{\alpha} \\
+\left(\delta_{1} \pi_{L}+\delta_{2} \pi_{M}\right)^{\alpha}+\left(\delta_{1} \xi_{L}+\delta_{2} \xi_{M}\right)^{\alpha} \\
\left.-\left(\delta_{1} \tau_{L}^{\alpha}+\delta_{2} \tau_{M}^{\alpha}\right)-\left(\delta_{1} v_{L}^{\alpha}+\delta_{2} v_{M}^{\alpha}\right) \delta_{1} \pi_{L}^{\alpha}+\delta_{2} \pi_{M}^{\alpha}\right)-\left(\delta_{1} \xi_{L}^{\alpha}+\delta_{2} \xi_{M}^{\alpha}\right)
\end{array}\right) .
\end{aligned}
$$

Now, we discuss a few properties of proposed measure introducing in Eq. (17).

\section{Particular Cases:}

Case 1. If $\alpha=1$ in Eq. (17), then

$\Phi^{* 1}(L, M)$
$=-\left(\begin{array}{c}\left(\delta_{1} \tau_{L}+\delta_{2} \tau_{M}\right) \log \left(\delta_{1} \tau_{L}+\delta_{2} \tau_{M}\right)+\left(\delta_{1} v_{L}+\delta_{2} v_{M}\right) \log \left(\delta_{1} v_{L}+\delta_{2} v_{M}\right) \\ +\left(\delta_{1} \pi_{L}+\delta_{2} \pi_{M}\right) \log \left(\delta_{1} \pi_{L}+\delta_{2} \pi_{M}\right)+\left(\delta_{1} \xi_{L}+\delta_{2} \xi_{M}\right) \log \left(\delta_{1} \xi_{L}+\delta_{2} \xi_{M}\right) \\ -\left(\delta_{1} \tau_{L} \log \tau_{L}+\delta_{2} \tau_{M} \log \tau_{M}\right)-\left(\delta_{1} v_{L} \log v_{L}+\delta_{2} v_{M} \log v_{M}\right) \\ -\left(\delta_{1} \pi_{L} \log \pi_{L}+\delta_{2} \pi_{M} \log \pi_{M}\right)-\left(\delta_{1} \xi_{L} \log \xi_{L}+\delta_{2} \xi_{M} \log \xi_{M}\right)\end{array}\right)$

It becomes a picture fuzzy dissimilarity measure studied by Lin (1991).

Case 2. If $\alpha=1, \delta_{1}=\delta_{2}=\frac{1}{2}$ in Eq. (17), then $\Phi^{* 1}(L, M)=$

$$
\left(\begin{array}{c}
\frac{1}{2}\left(\tau_{L}+\tau_{M}\right) \log \left(\frac{\tau_{L}+\tau_{M}}{2}\right)+\frac{1}{2}\left(v_{L}+v_{M}\right) \log \left(\frac{v_{L}+v_{M}}{2}\right) \\
+\frac{1}{2}\left(\pi_{L}+\pi_{M}\right) \log \left(\frac{\pi_{L}+\pi_{M}}{2}\right)+\frac{1}{2}\left(\xi_{L}+\xi_{M}\right) \log \left(\frac{\xi_{L}+\xi_{M}}{2}\right) \\
-\frac{1}{2}\left(\tau_{L} \log \tau_{L}+\tau_{M} \log \tau_{M}\right)-\frac{1}{2}\left(v_{L} \log v_{L}+v_{M} \log v_{M}\right) \\
-\frac{1}{2}\left(\pi_{L} \log \pi_{L}+\pi_{M} \log \pi_{M}\right)-\frac{1}{2}\left(\xi_{L} \log \xi_{L}+\xi_{M} \log \xi_{M}\right)
\end{array}\right) .
$$

It becomes a picture fuzzy dissimilarity measure for assigning the equal weights for each probability distribution.

Case 3. If $\delta_{1}=\delta_{2}=\frac{1}{2}$ in Eq. (17), then 


$$
\Phi^{* \alpha}(L, M)=\frac{1}{(1-\alpha)}\left(\begin{array}{c}
\left(\frac{\left(\tau_{L}+\tau_{M}\right)}{2}\right)^{\alpha}+\left(\frac{\left(v_{L}+v_{M}\right)}{2}\right)^{\alpha} \\
+\left(\frac{\left(\pi_{L}+\pi_{M}\right)}{2}\right)^{\alpha}+\left(\frac{\left(\xi_{L}+\xi_{M}\right)}{2}\right)^{\alpha} \\
-\frac{1}{2}\left(\tau_{L}^{\alpha}+\tau_{M}^{\alpha}\right)-\frac{1}{2}\left(v_{L}^{\alpha}+v_{M}^{\alpha}\right) \\
\frac{1}{2}\left(\pi_{L}^{\alpha}+\pi_{M}^{\alpha}\right)-\frac{1}{2}\left(\xi_{L}^{\alpha}+\xi_{M}^{\alpha}\right)
\end{array}\right) .
$$

It becomes a picture fuzzy dissimilarity measure corresponding to intuitionistic fuzzy dissimilarity measure studied by Hung and Yang (2006).

Case 4. If $\alpha=2$ in Eq. (17), then

$$
\begin{aligned}
& \Phi^{* 2}(L, M) \\
& =-\left(\begin{array}{c}
\left(\delta_{1} \tau_{L}+\delta_{2} \tau_{M}\right)^{2}+\left(\delta_{1} v_{L}+\delta_{2} v_{M}\right)^{2} \\
+\left(\delta_{1} \pi_{L}+\delta_{2} \pi_{M}\right)^{2}+\left(\delta_{1} \xi_{L}+\delta_{2} \xi_{M}\right)^{2} \\
\left.-\left(\delta_{1} \tau_{L}^{2}+\delta_{2} \tau_{M}^{2}\right)-\left(\delta_{1} v_{L}^{2}+\delta_{2} v_{M}^{2}\right) \delta_{1} \pi_{L}^{2}+\delta_{2} \pi_{M}^{2}\right)-\left(\delta_{1} \xi_{L}^{2}+\delta_{2} \xi_{M}^{2}\right)
\end{array}\right)
\end{aligned}
$$

which is based on Ginni index of diversity for the case of picture fuzzy sets.

Case 5. If $\alpha=2$ and $\delta_{1}=\delta_{2}=\frac{1}{2}$ in Eq. (17), then $\Phi^{* 2}(L, M)$

$$
\begin{aligned}
& =\frac{\left(\tau_{L}-\tau_{M}\right)^{2}+\left(v_{L}-v_{M}\right)^{2}+\left(\pi_{L}-\pi_{M}\right)^{2}+\left(\xi_{L}-\xi_{M}\right)^{2}}{4} \\
& =\frac{\left(g_{P F S}^{l}(L, M)\right)^{2}}{2}
\end{aligned}
$$

where $g_{P F S}^{l}(L, M)$ was characterize as a distance between PFSs. Hence, we can write as

$g_{P F S}^{l}(L, M)=\sqrt{2 \times \Phi^{* 2}(L, M)}$.

Case 6. If $\alpha=1, \xi_{L}=\xi_{M}=0$ in Eq. (19), then

$$
\begin{aligned}
& \Phi^{* 1}(L, M) \\
& =\left(\begin{array}{c}
\frac{1}{2}\left(\tau_{L}+\tau_{M}\right) \log \left(\frac{\tau_{L}+\tau_{M}}{2}\right)+\frac{1}{2}\left(v_{L}+v_{M}\right) \log \left(\frac{v_{L}+v_{M}}{2}\right) \\
+\frac{1}{2}\left(\pi_{L}+\pi_{M}\right) \log \left(\frac{\pi_{L}+\pi_{M}}{2}\right)-\frac{1}{2}\left(\tau_{L} \log \tau_{L}+\tau_{M} \log \tau_{M}\right) \\
\text { frac12( } \left.v_{L} \log v_{L}+v_{M} \log v_{M}\right)-\frac{1}{2}\left(\pi_{L} \log \pi_{L}+\pi_{M} \log \pi_{M}\right)
\end{array}\right)
\end{aligned}
$$

which becomes an intuitionistic fuzzy dissimilarity measure.

Case 7. If $\xi_{L}=\xi_{M}=0$ in Eq. (20), then

$$
\begin{aligned}
& \Phi^{* \alpha}(L, M)=\frac{1}{(1-\alpha)} \\
& \left(\begin{array}{c}
\left(\frac{\left(\tau_{L}+\tau_{M}\right)}{2}\right)^{\alpha}+\left(\frac{\left(v_{L}+v_{M}\right)}{2}\right)^{\alpha}+\left(\frac{\left(\pi_{L}+\pi_{M}\right)}{2}\right)^{\alpha} \\
-\frac{1}{2}\left(\tau_{L}^{\alpha}+\tau_{M}^{\alpha}\right)-\frac{1}{2}\left(v_{L}^{\alpha}+v_{M}^{\alpha}\right)-\frac{1}{2}\left(\pi_{L}^{\alpha}+\pi_{M}^{\alpha}\right)
\end{array}\right) .
\end{aligned}
$$

It becomes an intuitionistic fuzzy dissimilarity measure, which is studied by Hung and Yang (2006).

Case 8. If $\xi_{L}=\xi_{M}=0$ in Eq. (21), then

$$
\begin{aligned}
& \Phi^{* 2}(L, M) \\
& =-\left(\begin{array}{c}
\left(\delta_{1} \tau_{L}+\delta_{2} \tau_{M}\right)^{2}+\left(\delta_{1} v_{L}+\delta_{2} v_{M}\right)^{2}+\left(\delta_{1} \pi_{L}+\delta_{2} \pi_{M}\right)^{2} \\
-\left(\delta_{1} \tau_{L}^{2}+\delta_{2} \tau_{M}^{2}\right)-\left(\delta_{1} v_{L}^{2}+\delta_{2} v_{M}^{2}\right)-\left(\delta_{1} \pi_{L}^{2}+\delta_{2} \pi_{M}^{2}\right)
\end{array}\right)
\end{aligned}
$$

which is based on Ginni index of diversity for the case of intuitionistic fuzzy sets.

Case 9. If $\xi_{L}=\xi_{M}=0$ in Eq. (22), then

$$
\begin{aligned}
& \Phi^{* 2}(L, M)=\frac{\left(\tau_{L}-\tau_{M}\right)^{2}+\left(v_{L}-v_{M}\right)^{2}+\left(\pi_{L}-\pi_{M}\right)^{2}}{4} \\
& =\frac{\left(g_{I F S}^{l}(L, M)\right)^{2}}{2},
\end{aligned}
$$

where $g_{I F S}^{l}(L, M)$ was characterize as a distance between IFSs by Szmidt and Kacprzyk (2000). Hence, we can write as

$g_{I F S}^{l}(L, M)=\sqrt{2 \times \Phi^{* 2}(L, M)}$.

Case 10. If hesitancy degree, i.e., $\pi_{L}=\pi_{M}=0$ in Eq. (23), then

$$
\begin{aligned}
& \Phi^{* 1}(L, M) \\
& =\left(\begin{array}{l}
\frac{1}{2}\left(\tau_{L}+\tau_{M}\right) \log \left(\frac{\tau_{L}+\tau_{M}}{2}\right)+\frac{1}{2}\left(2-\tau_{L}-\tau_{M}\right) \log \left(\frac{2-\tau_{L}-\tau_{M}}{2}\right) \\
-\frac{1}{2}\left(\tau_{L} \log \tau_{L}+\tau_{M} \log \tau_{M}\right)-\frac{1}{2}\left(\left(1-\tau_{L}\right) \log \left(1-\tau_{L}\right)+\left(1-\tau_{M}\right) \log \left(1-\tau_{M}\right)\right)
\end{array}\right)
\end{aligned}
$$

which becomes Jensen-Shannon fuzzy divergence measure corresponding to Eq. (7).

Case 11. If hesitancy degree, i.e., $\pi_{L}=\pi_{M}=0$ in Eq. (24), then

$$
\begin{aligned}
& \Phi^{* \alpha}(L, M)=\frac{1}{(1-\alpha)} \\
& \left(\begin{array}{c}
\left(\frac{\tau_{L}+\tau_{M}}{2}\right)^{\alpha}+\left(\frac{\left(2-\tau_{L}-\tau_{M}\right)}{2}\right)^{\alpha} \\
-\frac{1}{2}\left(\tau_{L}^{\alpha}+\tau_{M}^{\alpha}\right)-\frac{1}{2}\left(\left(1-\tau_{L}\right)^{\alpha}+\left(1-\tau_{M}\right)^{\alpha}\right)
\end{array}\right)
\end{aligned}
$$

which becomes a fuzzy divergence measure corresponding to Eq. (8). 
Case 12. If hesitancy degree, i.e., $\pi_{L}=\pi_{M}=0$ in Eq. (25), then

$$
\begin{aligned}
& \Phi^{* 2}(L, M) \\
& =-\left(\begin{array}{c}
\left(\delta_{1} \tau_{L}+\delta_{2} \tau_{M}\right)^{2}+\left(\delta_{1}\left(1-\tau_{L}\right)+\delta_{2}\left(1-\tau_{M}\right)\right)^{2} \\
-\left(\delta_{1} \tau_{L}^{2}+\delta_{2} \tau_{M}^{2}\right)-\left(\delta_{1}\left(1-\tau_{L}\right)^{2}+\delta_{2}\left(1-\tau_{M}\right)^{2}\right)
\end{array}\right)
\end{aligned}
$$

which becomes a Jensen-Shannon J-divergence measure for fuzzy sets corresponding to Eq. (6).

Case 13. If hesitancy degree, i.e., $\pi_{L}=0$ and $\pi_{M}=0$ in Eq. (26), then

$$
\Phi^{* 2}(L, M)=\frac{\left(\tau_{L}-\tau_{M}\right)^{2}}{4}=\frac{\left(g_{F S}(L, M)\right)^{2}}{4} .
$$

Hence, we can write as

$$
g_{F S}(L, M)=\sqrt{4 \times \Phi^{* 2}(L, M)}
$$

which is characterize as an Euclidean distance between two fuzzy sets (FSs).

Presently, the justification of Eq. (17) is establishing through a theorem.

Theorem 3.1 Let $L, M \in P F S s(X)$, then $\Phi^{* \alpha}(L, M)$ satisfies the properties as follows:

1. $\Phi^{* \alpha}(L, M) \geq 0$ and as $\Phi^{* \alpha}(L, M)=0$ if and only if $L=M$.

2. Let $L, M, N \in P F S s(X)$ and $L \subseteq M \subseteq N$. Then $\Phi^{* \alpha}(L, M) \leq \Phi^{* \alpha}(L, N)$ and $\Phi^{* \alpha}(M, N) \leq \Phi^{* \alpha}(L, N)$.

\section{Proof}

1. Property 1 is directly from Jensen inequality.

2. Since $\Phi^{* \alpha}(L, M)$ is a convex function for $\alpha>0(\neq 1)$, therefore, $\Phi^{* \alpha}(L, M)$ increases with increase in $\| L-$ $M \|_{1}$ for all $\alpha>0(\neq 1)$ where

$$
|| L-M||_{1}=\left|\tau_{L}-\tau_{M}\right|+\left|v_{L}-v_{M}\right|+\left|\pi_{L}+\pi_{M}\right|+\left|\xi_{L}+\xi_{M}\right| .
$$

Let $L, M, N$ be PFSs in $X$ and $L \subseteq M \subseteq N$, then there are $\quad \tau_{L} \leq \tau_{M} \leq \tau_{N}, \quad v_{L} \leq v_{M} \leq v_{N}, \quad \pi_{L} \leq \pi_{M} \leq \pi_{N}$. Then, we have

$$
\|L-M\|_{1} \leq\|L-N\|_{1}
$$

and

$$
\|M-N\|_{1} \leq\|L-N\|_{1} .
$$

Thus, $\quad \Phi^{* \alpha}(L, M) \leq \Phi^{* \alpha}(L, N) \quad$ and $\quad \Phi^{* \alpha}(M, N)$ $\leq \Phi^{* \alpha}(L, N)$.

Definition $3.6\left(R_{\text {new }}^{\alpha}(L, M)\right.$ for Finite Universal Set $)$ : Now, we extend the concept of Jensen-Tsallis divergence measure from single element universe to finite universal set. For this, for coefficient weights $\delta_{1}, \delta_{2}$, let $X=$ $\left\{t_{1}, t_{2}, \ldots, t_{k}\right\}$ be a fixed set and for any $L, M \in P F S s(X)$, corresponding to (17), we present a generalized JensenTsallis picture fuzzy divergence $R_{\text {new }}^{\alpha}(L, M)$ as

$$
\begin{gathered}
R_{\text {new }}^{\alpha}(L, M)=\frac{1}{k} \sum_{p=1}^{k} \Phi^{* \alpha}(L, M) \\
\left.(1-\alpha) k \begin{array}{c}
1 \\
\left(\delta_{1} \tau_{L}+\delta_{2} \tau_{M}\right)^{\alpha}+\left(\delta_{1} v_{L}+\delta_{2} v_{M}\right)^{\alpha} \\
+\left(\delta_{1} \pi_{L}+\delta_{2} \pi_{M}\right)^{\alpha}+\left(\delta_{1} \xi_{L}+\delta_{2} \xi_{M}\right)^{\alpha} \\
\left.-\left(\delta_{1} \tau_{L}^{\alpha}+\delta_{2} \tau_{M}^{\alpha}\right)-\left(\delta_{1} v_{L}^{\alpha}+\delta_{2} v_{M}^{\alpha}\right) \delta_{1} \pi_{L}^{\alpha}+\delta_{2} \pi_{M}^{\alpha}\right)-\left(\delta_{1} \xi_{L}^{\alpha}+\delta_{2} \xi_{M}^{\alpha}\right)
\end{array}\right) .
\end{gathered}
$$

Now, we give some properties of measure (31) through a theorem.

Theorem 3.2 For $L, M, I \in \operatorname{PFSs}(X)$,

1. $R_{\text {new }}^{\alpha}(L, M)=0$ iff $L=M$;

2. $R_{\text {new }}^{\alpha}\left(L, L^{c}\right)=0$ iff $\tau_{L}^{c}(t)=1-\tau_{L}(t)$ for all $t \in X$;

3. $R_{\text {new }}^{\alpha}(L, L \cup M)=R_{\text {new }}^{\alpha}(L \cap M, M) \leq R_{\text {new }}^{\alpha}(L, M)$;

4. $R_{\text {new }}^{\alpha}(L \cup M, L \cap M)=R_{\text {new }}^{\alpha}(L \cap M, L \cup M)$ $=R_{\text {new }}^{\alpha}(L, M)$;

5. $R_{\text {new }}^{\alpha}(L \cap M, L \cup M)=R_{\text {new }}^{\alpha}(M, L)$;

6. $R_{\text {new }}^{\alpha}(L, L \cup M)+R_{\text {new }}^{\alpha}(L, L \cap M)=R_{\text {new }}^{\alpha}(L, M)$;

7. $R_{\text {new }}^{\alpha}(M, L \cup M)+R_{\text {new }}^{\alpha}(M, L \cap M)=R_{\text {new }}^{\alpha}(M, L)$;

8. $R_{\text {new }}^{\alpha}(L \cup M, I) \leq R_{\text {new }}^{\alpha}(L, I)+R_{\text {new }}^{\alpha}(M, I)$;

9. $R_{\text {new }}^{\alpha}(L \cap M, I) \leq R_{\text {new }}^{\alpha}(L, I)+R_{\text {new }}^{\alpha}(M, I)$;

10. $R_{\text {new }}^{\alpha}(L \cup M, I)+R_{\text {new }}^{\alpha}(L \cap M, I)$ $=R_{\text {new }}^{\alpha}(L, I)+R_{\text {new }}^{\alpha}(M, I)$;

11. $R_{\text {new }}^{\alpha}(L, M)=R_{\text {new }}^{\alpha}\left(L^{c}, M^{c}\right)$;

12. $R_{\text {new }}^{\alpha}\left(L, M^{c}\right)=R_{\text {new }}^{\alpha}\left(L^{c}, M\right)$;

13. $R_{\text {new }}^{\alpha}(L, M)+R_{\text {new }}^{\alpha}\left(L^{c}, M\right)$ $=R_{\text {new }}^{\alpha}\left(L^{c}, M^{c}\right)+R_{\text {new }}^{\alpha}\left(L, M^{c}\right)$; where $L^{c}$ and $M^{c}$ are the complements of PFSs $L$ and $M$, respectively.

Proof Suppose $X$ bifurcate into two parts $X_{1}$ and $X_{2}$ such that:

$$
X_{1}=\{t \in X \mid L(t) \subseteq M(t)\},
$$

and $\quad X_{2}=\{t \in X \mid L(t) \supseteq M(t)\}$.

From (32), it is clear that for all $t \in X_{1}$,

$$
\tau_{L}(t) \leq \tau_{M}(t) \quad \text { and } \quad v_{L}(t) \geq v_{M}(t) \quad \text { and } \quad \pi_{L}(t) \geq \pi_{M}(t)
$$

and for all $t \in X_{2}$,

$\tau_{L}(t) \geq \tau_{M}(t) \quad$ and $\quad v_{L}(t) \leq \tau_{M}(t) \quad$ and $\quad \pi_{L}(t) \leq \pi_{M}(t)$.

1. Proof of properties (1-2) is directly from the definition (31). 
3. Since

$$
\begin{aligned}
& R_{\text {new }}^{\alpha}(L, L \cup M)=\frac{1}{(1-\alpha) k} \sum_{p=1}^{k} \\
& \left(\begin{array}{c}
\left(\delta_{1} \tau_{L}+\delta_{2} \tau_{L \cup M}\right)^{\alpha}+\left(\delta_{1} v_{L}+\delta_{2} v_{L \cup M}\right)^{\alpha} \\
+\left(\delta_{1} \pi_{L}+\delta_{2} \pi_{L \cup M}\right)^{\alpha}+\left(\delta_{1} \xi_{L}+\delta_{2} \xi_{L \cup M}\right)^{\alpha} \\
-\left(\delta_{1} \tau_{L}^{\alpha}+\delta_{2} \tau_{L \cup M}^{\alpha}\right)-\left(\delta_{1} v_{L}^{\alpha}+\delta_{2} v_{L \cup M}^{\alpha}\right) \\
-\left(\delta_{1} \pi_{L}^{\alpha}+\delta_{2} \pi_{L \cup M}^{\alpha}\right)-\left(\delta_{1} \xi_{L}^{\alpha}+\delta_{2} \xi_{L \cup M}^{\alpha}\right)
\end{array}\right)
\end{aligned}
$$

This implies

$R_{\text {new }}^{\alpha}(L, L \cup M)=$

$\sum_{X_{1}}\left(\begin{array}{c}\left(\delta_{1} \tau_{L}+\delta_{2} \tau_{M}\right)^{\alpha}+\left(\delta_{1} v_{L}+\delta_{2} v_{M}\right)^{\alpha}+\left(\delta_{1} \pi_{L}+\delta_{2} \pi_{M}\right)^{\alpha}+\left(\delta_{1} \xi_{L}+\delta_{2} \xi_{M}\right)^{\alpha} \\ -\left(\delta_{1} \tau_{L}^{\alpha}+\delta_{2} \tau_{M}^{\alpha}\right)-\left(\delta_{1} v_{L}^{\alpha} \delta_{2} v_{M}^{\alpha}\right)-\left(\delta_{1} \pi_{L}^{\alpha}+\delta_{2} \pi_{M}^{\alpha}\right)-\left(\delta_{1} \xi_{L}^{\alpha}+\delta_{2} \xi_{M}^{\alpha}\right)\end{array}\right)$

$$
\begin{aligned}
& +\sum_{X_{2}}\left(\begin{array}{c}
\left(\delta_{1} \tau_{L}+\delta_{2} \tau_{L}\right)^{\alpha}+\left(\delta_{1} v_{L}+\delta_{2} v_{L}\right)^{\alpha}+\left(\delta_{1} \pi_{L}+\delta_{2} \pi_{L}\right)^{\alpha}+\left(\delta_{1} \xi_{L}+\delta_{2} \xi_{L}\right)^{\alpha} \\
-\left(\delta_{1} \tau_{L}^{\alpha}+\delta_{2} \tau_{L}^{\alpha}\right)-\left(\delta_{1} v_{L}^{\alpha}+\delta_{2} v_{L}^{\alpha}\right)-\left(\delta_{1} \pi_{L}^{\alpha}+\delta_{2} \pi_{L}^{\alpha}\right)-\left(\delta_{1} \xi_{L}^{\alpha}+\delta_{2} \xi_{L}^{\alpha}\right)
\end{array}\right) \\
& \leq \sum_{p=1}^{k}\left(\begin{array}{c}
\left(\delta_{1} \tau_{L}+\delta_{2} \tau_{L \cup M}\right)^{\alpha}+\left(\delta_{1} v_{L}+\delta_{2} v_{L \cup M}\right)^{\alpha} \\
+\left(\delta_{1} \pi_{L}+\delta_{2} \pi_{L \cup M}\right)^{\alpha}+\left(\delta_{1} \xi_{L}+\delta_{2} \xi_{L \cup M}\right)^{\alpha} \\
-\left(\delta_{1} \tau_{L}^{\alpha}+\delta_{2} \tau_{L \cup M}^{\alpha}\right)-\left(\delta_{1} v_{L}^{\alpha}+\delta_{2} v_{L \cup M}^{\alpha}\right) \\
-\left(\delta_{1} \pi_{L}^{\alpha}+\delta_{2} \pi_{L \cup M}^{\alpha}\right)-\left(\delta_{1} \xi_{L}^{\alpha}+\delta_{2} \xi_{L \cup M}^{\alpha}\right)
\end{array}\right)=R_{\text {new }}^{\alpha}(L, M)
\end{aligned}
$$

Similarly, we can prove that $R_{\text {new }}^{\alpha}(L \cap M, M) \leq R_{\text {new }}^{\alpha}(L, M)$.

4. From property 4 , we have

$$
\begin{aligned}
R_{\text {new }}^{\alpha}(L \cup M, L \cap M) & =\sum_{X_{1}} R_{\text {new }}^{\alpha}(M, L)+\sum_{X_{2}} R_{\text {new }}^{\alpha}(L, M) \\
& =R_{\text {new }}^{\alpha}(L, M),
\end{aligned}
$$

similarly, we can prove that

$$
\begin{aligned}
R_{\text {new }}^{\alpha}(L \cap M, L \cup M) & =\sum_{X_{1}} R_{\text {new }}^{\alpha}(M, L)+\sum_{X_{2}} R_{\text {new }}^{\alpha}(L, M) \\
& =R_{\text {new }}^{\alpha}(L, M) .
\end{aligned}
$$

5. Property 5 is proved as same as of the property 4 . 6. Consider

$$
\begin{aligned}
& R_{\text {new }}^{\alpha}(L, L \cup M)+R_{\text {new }}^{\alpha}(L, L \cap M) \\
& =\sum_{X_{1}} R_{\text {new }}^{\alpha}(L, M)+\sum_{X_{2}} R_{\text {new }}^{\alpha}(L, L) \\
& \quad+\sum_{X_{1}} R_{\text {new }}^{\alpha}(L, L)+\sum_{X_{2}} R_{\text {new }}^{\alpha}(L, M) \\
& =R_{\text {new }}^{\alpha}(L, M) .
\end{aligned}
$$

7. Property 7 is proved as same as the property 6 .
8. Consider

$$
\begin{aligned}
& R_{\text {new }}^{\alpha}(L, I)+R_{\text {new }}^{\alpha}(M, I)-R_{\text {new }}^{\alpha}(L \cup M, I) \\
& =\frac{1}{(1-\alpha) k} \sum_{p=1}^{k}\left(\begin{array}{c}
\left(\delta_{1} \tau_{L}+\delta_{2} \tau_{I}\right)^{\alpha}+\left(\delta_{1} v_{L}+\delta_{2} v_{I}\right)^{\alpha} \\
+\left(\delta_{1} \pi_{L}+\delta_{2} \pi_{I}\right)^{\alpha}+\left(\delta_{1} \xi_{L}+\delta_{2} \xi_{I}\right)^{\alpha} \\
-\left(\delta_{1} \tau_{L}^{\alpha}+\delta_{2} \tau_{I}^{\alpha}\right)-\left(\delta_{1} v_{L}^{\alpha}+\delta_{2} v_{I}^{\alpha}\right) \\
\left.-\delta_{1} \pi_{L}^{\alpha}+\delta_{2} \pi_{I}^{\alpha}\right)-\left(\delta_{1} \xi_{L}^{\alpha}+\delta_{2} \xi_{I}^{\alpha}\right)
\end{array}\right) \\
& +\frac{1}{(1-\alpha) k} \sum_{p=1}^{k}\left(\begin{array}{c}
\left(\delta_{1} \tau_{M}+\delta_{2} \tau_{I}\right)^{\alpha}+\left(\delta_{1} v_{M}+\delta_{2} v_{I}\right)^{\alpha} \\
+\left(\delta_{1} \pi_{M}+\delta_{2} \pi_{I}\right)^{\alpha}+\left(\delta_{1} \xi_{M}+\delta_{2} \xi_{I}\right)^{\alpha} \\
-\left(\delta_{1} \tau_{M}^{\alpha}+\delta_{2} \tau_{I}^{\alpha}\right)-\left(\delta_{1} v_{M}^{\alpha}+\delta_{2} v_{I}^{\alpha}\right) \\
\left.-\delta_{1} \pi_{M}^{\alpha}+\delta_{2} \pi_{I}^{\alpha}\right)-\left(\delta_{1} \xi_{M}^{\alpha}+\delta_{2} \xi_{I}^{\alpha}\right)
\end{array}\right) \\
& -\frac{1}{(1-\alpha) k} \sum_{p=1}^{k}\left(\begin{array}{c}
\left(\delta_{1} \tau_{L \cup M}+\delta_{2} \tau_{I}\right)^{\alpha}+\left(\delta_{1} v_{L \cup M}+\delta_{2} v_{I}\right)^{\alpha} \\
+\left(\delta_{1} \pi_{L \cup M}+\delta_{2} \pi_{I}\right)^{\alpha}+\left(\delta_{1} \xi_{L \cup M}+\delta_{2} \xi_{I}\right)^{\alpha} \\
-\left(\delta_{1} \tau_{L \cup M}^{\alpha}+\delta_{2} \tau_{I}^{\alpha}\right)-\left(\delta_{1} v_{L \cup M}^{\alpha}+\delta_{2} v_{I}^{\alpha}\right) \\
\left.-\delta_{1} \pi_{L \cup M}^{\alpha}+\delta_{2} \pi_{I}^{\alpha}\right)-\left(\delta_{1} \xi_{L \cup M}^{\alpha}+\delta_{2} \xi_{I}^{\alpha}\right)
\end{array}\right)
\end{aligned}
$$

This implies $R_{\text {new }}^{\alpha}(L, I)+R_{\text {new }}^{\alpha}(M, I)-R_{\text {new }}^{\alpha}(L \cup M, I)$

$$
\begin{gathered}
=\frac{1}{(1-\alpha) k} \sum_{p=1}^{k}\left(\begin{array}{c}
\left(\delta_{1} \tau_{L}+\delta_{2} \tau_{I}\right)^{\alpha}+\left(\delta_{1} v_{L}+\delta_{2} v_{I}\right)^{\alpha} \\
+\left(\delta_{1} \pi_{L}+\delta_{2} \pi_{I}\right)^{\alpha}+\left(\delta_{1} \xi_{L}+\delta_{2} \xi_{I}\right)^{\alpha} \\
-\left(\delta_{1} \tau_{L}^{\alpha}+\delta_{2} \tau_{I}^{\alpha}\right)-\left(\delta_{1} v_{L}^{\alpha}+\delta_{2} v_{I}^{\alpha}\right) \\
\left.-\delta_{1} \pi_{L}^{\alpha}+\delta_{2} \pi_{I}^{\alpha}\right)-\left(\delta_{1} \xi_{L}^{\alpha}+\delta_{2} \xi_{I}^{\alpha}\right)
\end{array}\right) \\
+\frac{1}{(1-\alpha) k} \sum_{p=1}^{k}\left(\begin{array}{c}
\left(\delta_{1} \tau_{M}+\delta_{2} \tau_{I}\right)^{\alpha}+\left(\delta_{1} v_{M}+\delta_{2} v_{I}\right)^{\alpha} \\
+\left(\delta_{1} \pi_{M}+\delta_{2} \pi_{I}\right)^{\alpha}+\left(\delta_{1} \xi_{M}+\delta_{2} \xi_{I}\right)^{\alpha} \\
-\left(\delta_{1} \tau_{M}^{\alpha}+\delta_{2} \tau_{I}^{\alpha}\right)-\left(\delta_{1} v_{M}^{\alpha}+\delta_{2} v_{I}^{\alpha}\right) \\
\left.-\delta_{1} \pi_{M}^{\alpha}+\delta_{2} \pi_{I}^{\alpha}\right)-\left(\delta_{1} \xi_{M}^{\alpha}+\delta_{2} \xi_{I}^{\alpha}\right)
\end{array}\right) \\
-\frac{1}{(1-\alpha) k} \sum_{X_{1}}\left(\begin{array}{c}
\left(\delta_{1} \tau_{M}+\delta_{2} \tau_{I}\right)^{\alpha}+\left(\delta_{1} v_{M}+\delta_{2} v_{I}\right)^{\alpha} \\
+\left(\delta_{1} \pi_{M}+\delta_{2} \pi_{I}\right)^{\alpha}+\left(\delta_{1} \xi_{M}+\delta_{2} \xi_{I}\right)^{\alpha} \\
-\left(\delta_{1} \tau_{M}^{\alpha}+\delta_{2} \tau_{I}^{\alpha}\right)-\left(\delta_{1} v_{M}^{\alpha}+\delta_{2} v_{I}^{\alpha}\right) \\
\left.-\delta_{1} \pi_{M}^{\alpha}+\delta_{2} \pi_{I}^{\alpha}\right)-\left(\delta_{1} \xi_{M}^{\alpha}+\delta_{2} \xi_{I}^{\alpha}\right)
\end{array}\right) \\
-\frac{1}{(1-\alpha) k} \sum_{X_{2}}\left(\begin{array}{c}
\left(\delta_{1} \tau_{L}+\delta_{2} \tau_{I}\right)^{\alpha}+\left(\delta_{1} v_{L}+\delta_{2} v_{I}\right)^{\alpha} \\
+\left(\delta_{1} \pi_{L}+\delta_{2} \pi_{I}\right)^{\alpha}+\left(\delta_{1} \xi_{L}+\delta_{2} \xi_{I}\right)^{\alpha} \\
-\left(\delta_{1} \tau_{L}^{\alpha}+\delta_{2} \tau_{I}^{\alpha}\right)-\left(\delta_{1} v_{L}^{\alpha}+\delta_{2} v_{I}^{\alpha}\right) \\
\left.-\delta_{1} \pi_{L}^{\alpha}+\delta_{2} \pi_{I}^{\alpha}\right)-\left(\delta_{1} \xi_{L}^{\alpha}+\delta_{2} \xi_{I}^{\alpha}\right)
\end{array}\right) \geq 0 .
\end{gathered}
$$

Therefore, $R_{\text {new }}^{\alpha}(L \cup M, I) \leq R_{\text {new }}^{\alpha}(L, I)+R_{\text {new }}^{\alpha}(M, I)$.

9. Proof of property 9 simply follows from property 8 .

10. Consider $R_{\text {new }}^{\alpha}(L \cup M, I)+R_{\text {new }}^{\alpha}(L \cap M, I)$

$$
\begin{gathered}
=\frac{1}{(1-\alpha) k} \sum_{p=1}^{k} \begin{array}{c}
\left(\delta_{1} \tau_{L \cup M}+\delta_{2} \tau_{I}\right)^{\alpha}+\left(\delta_{1} v_{L \cup M}+\delta_{2} v_{I}\right)^{\alpha} \\
+\left(\delta_{1} \pi_{L \cup M}+\delta_{2} \pi_{I}\right)^{\alpha}+\left(\delta_{1} \xi_{L \cup M}+\delta_{2} \xi_{I}\right)^{\alpha}
\end{array} \\
\left(\begin{array}{c}
\left.+\left(\delta_{1} \tau_{L \cup M}^{\alpha}+\delta_{2} \tau_{I}^{\alpha}\right)-\left(\delta_{1} v_{L \cup M}^{\alpha}+\delta_{2} v_{I}^{\alpha}\right) \delta_{1} \pi_{L \cup M}^{\alpha}+\delta_{2} \pi_{I}^{\alpha}\right)-\left(\delta_{1} \xi_{L \cup M}^{\alpha}+\delta_{2} \xi_{I}^{\alpha}\right)
\end{array}\right) \\
\left.+\frac{1}{(1-\alpha) k} \sum_{p=1}^{k} \begin{array}{c}
\left(\delta_{1} \tau_{L \cap M}+\delta_{2} \tau_{I}\right)^{\alpha}+\left(\delta_{1} v_{L \cap M}+\delta_{2} v_{I}\right)^{\alpha} \\
+\left(\delta_{1} \pi_{L \cap M}+\delta_{2} \pi_{I}\right)^{\alpha}+\left(\delta_{1} \xi_{L \cap M}+\delta_{2} \xi_{I}\right)^{\alpha} \\
\left.-\left(\delta_{1} \tau_{L \cap M}^{\alpha}+\delta_{2} \tau_{I}^{\alpha}\right)-\left(\delta_{1} v_{L \cap M}^{\alpha}+\delta_{2} v_{I}^{\alpha}\right) \delta_{1} \pi_{L \cap M}^{\alpha}+\delta_{2} \pi_{I}^{\alpha}\right)-\left(\delta_{1} \xi_{L \cap M}^{\alpha}+\delta_{2} \xi_{I}^{\alpha}\right)
\end{array}\right)
\end{gathered}
$$

Using (35), we obtain 


$$
\begin{aligned}
& =\sum_{X_{1}} R_{\text {new }}^{\alpha}(M, I)+\sum_{X_{2}} R_{\text {new }}^{\alpha}(L, I) \\
& +\sum_{X_{1}} R_{\text {new }}^{\alpha}(L, I)+\sum_{X_{2}} R_{\text {new }}^{\alpha}(M, I) \\
& =R_{\text {new }}^{\alpha}(L, I)+R_{\text {new }}^{\alpha}(M, I) .
\end{aligned}
$$

11. Property 11 is proved directly from definition (31). 12. Property 12 is proved directly from definition (31).

13. Property 13 is proved directly from definition (31).

\section{Applications of proposed picture fuzzy divergence measure}

Under this section, we present the applications for an outbreak of COVID-19 and pattern recognition.

\subsection{Novel coronavirus disease (NCOVID-19)}

To exhibit the relevance and legitimacy of the proposed method, we apply it on an outbreak of novel coronavirus ailment (NCOVID-19).

Example 1 Now, in the circumstance of COVID-19, it is fundamental to give a productive route in crisis reaction for evading extra misfortunes and to save the lives of the people. Because of such a crisis choice, the wellbeing specialists need to make a quick reaction, desperately salvage to control the circumstance proficiently and prevent it from more deaths. There are eight fundamental public health emergency factors to diminish the overall danger of this ailment. The best preventive measures are: let us consider the situations $\mathrm{Z}=\left\{A_{1}\right.$-Medical facilities, $A_{2}$-research needs, $A_{3}$-lock down the borders, $A_{4}$-banned intracity transportation, $A_{5}$-public awareness $\}$ and five emergency factor to reduce this risk $\mathrm{D}=\left\{\eta_{1}\right.$-clinical management (CM), $\eta_{2}$-monitoring (MN), $\eta_{3}$-country-level coordination (CLC), $\eta_{4}$-consult experts (CE), $\eta_{5}$-increase personal protective equipment (IPP) $\}$ with symptoms $\mathrm{V}=\left\{\rho_{1}\right.$-shortness of breath, $\rho_{2}$-chest pain, $\rho_{3}$-loss of taste, $\rho_{4}$-loss of speech, $\rho_{5}$-conjunctivitis $\}$. Every element in situations, symptoms and emergency factor is given as a picture fuzzy number. One need to find a proper preventive measure for every emergency situation in context of symptoms. The procedure is repeated for all components. At last, we recommend the measure for emergency situation whose symptoms have minimum degree of divergence measure. Let situations $A_{1}, A_{2}, A_{3}, A_{4}, A_{5}$, w.r.t. all symptoms in the form of following PFSs:

$$
\begin{gathered}
A_{1}=\left\{\left(t_{1}, 0.1,0.4,0.3,0.2\right),\left(t_{2}, 0.4,0.1,0.3,0.2\right),\left(t_{3}, 0.3,0.2,0.4,0.1\right),\right. \\
\left.\left(t_{4}, 0.2,0.3,0.5,0.0\right),\left(t_{5}, 0.5,0.1,0.3,0.1\right)\right\}, \\
A_{2}=\left\{\left(t_{1}, 0.3,0.3,0.3,0.1\right),\left(t_{2}, 0.0,0.2,0.7,0.1\right),\left(t_{3}, 0.6,0.1,0.1,0.2\right),\right. \\
\left.\left(t_{4}, 0.4,0.4,0.2,0.0\right),\left(t_{5}, 0.1,0.5,0.3,0.1\right)\right\}, \\
A_{3}=\left\{\left(t_{1}, 0.5,0.1,0.3,0.1\right),\left(t_{2}, 0.3,0.4,0.3,0.0\right),\left(t_{3}, 0.6,0.1,0.3,0.0\right),\right. \\
\left.\left(t_{4}, 0.4,0.4,0.1,0.1\right),\left(t_{5}, 0.1,0.5,0.2,0.2\right)\right\}, \\
A_{4}=\left\{\left(t_{1}, 0.6,0.2,0.1,0.1\right),\left(t_{2}, 0.3,0.2,0.4,0.1\right),\left(t_{3}, 0.4,0.2,0.3,0.1\right),\right. \\
\left.\left(t_{4}, 0.6,0.1,0.1,0.2\right),\left(t_{5}, 0.4,0.2,0.2,0.2\right)\right\}, \\
A_{5}=\left\{\left(t_{1}, 0.1,0.4,0.3,0.2\right),\left(t_{2}, 0.4,0.1,0.3,0.2\right),\left(t_{3}, 0.3,0.2,0.4,0.1\right),\right. \\
\left.\left(t_{4}, 0.2,0.3,0.4,0.1\right),\left(t_{5}, 0.5,0.1,0.3,0.1\right)\right\} .
\end{gathered}
$$

Each factor $\eta_{p}(p=1,2,3,4,5)$ w.r.t. all the symptoms can be seen in the form of PFSs as:

$$
\begin{gathered}
\eta_{1}=\left\{\left(t_{1}, 0.6,0.1,0.2,0.1\right),\left(t_{2}, 0.3,0.2,0.4,0.1\right),\left(t_{3}, 0.4,0.2,0.3,0.1\right),\right. \\
\left.\left(t_{4}, 0.5,0.2,0.1,0.2\right),\left(t_{5}, 0.4,0.2,0.2,0.2\right)\right\}, \\
\eta_{2}=\left\{\left(t_{1}, 0.5,0.3,0.1,0.1\right),\left(t_{2}, 0.3,0.4,0.3,0.0\right),\left(t_{3}, 0.7,0.2,0.1,0.0\right),\right. \\
\left.\left(t_{4}, 0.3,0.1,0.5,0.1\right),\left(t_{5}, 0.3,0.4,0.2,0.1\right)\right\}, \\
\eta_{3}=\left\{\left(t_{1}, 0.1,0.1,0.6,0.2\right),\left(t_{2}, 0.3,0.1,0.5,0.1\right),\left(t_{3}, 0.2,0.2,0.4,0.2\right),\right. \\
\left.\left(t_{4}, 0.4,0.4,0.1,0.1\right),\left(t_{5}, 0.3,0.2,0.4,0.1\right)\right\}, \\
\eta_{4}=\left\{\left(t_{1}, 0.4,0.1,0.3,0.2\right),\left(t_{2}, 0.5,0.2,0.3,0.0\right),\left(t_{3}, 0.4,0.0,0.3,0.3\right),\right. \\
\left.\left(t_{4}, 0.7,0.2,0.0,0.1\right),\left(t_{5}, 0.6,0.1,0.1,0.2\right)\right\}, \\
\eta_{5}=\left\{\left(t_{1}, 0.7,0.1,0.1,0.1\right),\left(t_{2}, 0.2,0.4,0.3,0.1\right),\left(t_{3}, 0.2,0.5,0.1,0.2\right),\right. \\
\left.\left(t_{4}, 0.1,0.2,0.5,0.2\right),\left(t_{5}, 0.3,0.3,0.3,0.1\right)\right\},
\end{gathered}
$$

Now, we calculate the values of situations with respect to symptoms for different values of $\delta_{1}, \delta_{2}$. Calculated values are given in Table 1, 2, 3, 2, 6 .

Notations: $\eta_{1}$-clinical management, $\eta_{2}$-monitoring, $\eta_{3}$ country level coordination, $\eta_{4}$-consult experts, $\eta_{5}$-increase personal protective equipment.

Abbreviations: In Table 7, some abbreviations are used like: CM-clinical management, MN-monitoring, CLCcountry level coordination, CE-consult experts, IPP-increase personal protective equipment.

We conclude the Tables 1, 2, 3, 4, 5, 6 in Table 7. In Table 7, proposed picture fuzzy divergence measure (31) suggests that the factors CLC, IPP, CM, CLC, MN are the

Table 1 Values for each considered situation for $\delta_{1}=.1, \delta_{2}=.9$

\begin{tabular}{llllll}
\hline & $\eta_{1}$ & $\eta_{2}$ & $\eta_{3}$ & $\eta_{4}$ & $\eta_{5}$ \\
\hline$A_{1}$ & 0.081 & 0.014 & $\mathbf{0 . 0 0 2}$ & 1.775 & 0.019 \\
$A_{2}$ & 0.019 & 1.098 & 0.554 & 0.521 & $\mathbf{0 . 0 1 8}$ \\
$A_{3}$ & $\mathbf{0 . 0 5 0}$ & 0.511 & 0.578 & 0.586 & 0.630 \\
$A_{4}$ & 0.071 & 0.011 & $\mathbf{0 . 0 1 0}$ & 1.221 & 0.025 \\
$A_{5}$ & 0.030 & $\mathbf{0 . 0 0 2}$ & 0.003 & 1.156 & 0.071 \\
Results: & $A_{1}\left(\eta_{3}\right)$ & $A_{2}\left(\eta_{5}\right)$ & $A_{3}\left(\eta_{1}\right)$ & $A_{4}\left(\eta_{3}\right)$ & $A_{5}\left(\eta_{2}\right)$
\end{tabular}

Bold indicates minimum value of each situation with respect to symptoms 
Table 2 Values for each considered situation for $\delta_{1}=.2, \delta_{2}=.8$

\begin{tabular}{llllll}
\hline & $\eta_{1}$ & $\eta_{2}$ & $\eta_{3}$ & $\eta_{4}$ & $\eta_{5}$ \\
\hline$A_{1}$ & 0.135 & 0.025 & $\mathbf{0 . 0 0 4}$ & 1.996 & 0.032 \\
$A_{2}$ & 0.034 & 1.238 & 0.619 & 0.605 & $\mathbf{0 . 0 3 2}$ \\
$A_{3}$ & $\mathbf{0 . 0 8 4}$ & 0.588 & 0.660 & 0.678 & 0.722 \\
$A_{4}$ & 0.118 & 0.020 & $\mathbf{0 . 0 1 8}$ & 1.138 & 0.043 \\
$A_{5}$ & 0.052 & $\mathbf{0 . 0 0 4}$ & 0.005 & 1.298 & 0.120 \\
Results: & $A_{1}\left(\eta_{3}\right)$ & $A_{2}\left(\eta_{5}\right)$ & $A_{3}\left(\eta_{1}\right)$ & $A_{4}\left(\eta_{3}\right)$ & $A_{5}\left(\eta_{2}\right)$ \\
\hline
\end{tabular}

Bold indicates minimum value of each situation with respect to symptoms

Table 3 Values for each considered situation for $\delta_{1}=.3, \delta_{2}=.7$

\begin{tabular}{llllll}
\hline & $\eta_{1}$ & $\eta_{2}$ & $\eta_{3}$ & $\eta_{4}$ & $\eta_{5}$ \\
\hline$A_{1}$ & 0.170 & 0.032 & $\mathbf{0 . 0 0 5}$ & 2.114 & 0.042 \\
$A_{2}$ & 0.043 & 1.311 & 0.652 & 0.652 & $\mathbf{0 . 0 4 1}$ \\
$A_{3}$ & $\mathbf{0 . 1 0 6}$ & 0.630 & 0.706 & 0.785 & 0.773 \\
$A_{4}$ & 0.149 & 0.027 & $\mathbf{0 . 0 2 4}$ & 1.466 & 0.056 \\
$A_{5}$ & 0.067 & $\mathbf{0 . 0 0 5}$ & 0.007 & 1.371 & 0.152 \\
Results: & $A_{1}\left(\eta_{3}\right)$ & $A_{2}\left(\eta_{5}\right)$ & $A_{3}\left(\eta_{1}\right)$ & $A_{4}\left(\eta_{3}\right)$ & $A_{5}\left(\eta_{2}\right)$ \\
\hline
\end{tabular}

Bold indicates minimum value of each situation with respect to symptoms

essential measures for the situations $A_{1}, A_{2}, A_{3}, A_{4}, A_{5}$ respectively for various values of parameters $\delta_{1}, \delta_{2}$, according to the principle of the minimum degree of divergence measure between PFSs. Thus, we have come to the conclusion that according to our proposed measure, above mentioned factors are the main preventive measures that decrease the overall risk of this infection. The results of proposed measure which are obtained on the different value of $\delta_{1}, \delta_{2}$ are depicted by Fig. 1 .

In Fig. 1, different colors show the different factors as first light blue color presents country-level coordination (CLC), orange color presents increase personal protective equipment (IPP), gray color presents clinical management (CM), yellow color presents country level coordination (CLC) and blue color presents monitoring. From Fig. 1, we can seen that the factors CLC, IPP, CM, CLC, MN are consistently occurring in a manner for various values of parameters $\delta_{1}, \delta_{2}$. This shows the consistency of the proposed measure.

\subsection{Comparison with other existing measures}

Under this subsection, we present the comparative study for demonstrating the usefulness of the proposed measure. Comparisons are taken among the picture fuzzy divergence measures suggested by: Hung and Yang (2006), Son et al. (2017), Ashraf et al. (2019), Wang et al. (2017), Son
Table 4 Values for each considered situation for $\delta_{1}=.4, \delta_{2}=.6$

\begin{tabular}{llllll}
\hline & $\eta_{1}$ & $\eta_{2}$ & $\eta_{3}$ & $\eta_{4}$ & $\eta_{5}$ \\
\hline$A_{1}$ & 0.190 & 0.037 & $\mathbf{0 . 0 0 6}$ & 2.170 & 0.048 \\
$A_{2}$ & 0.049 & 1.349 & 0.670 & 0.677 & $\mathbf{0 . 0 4 7}$ \\
$A_{3}$ & $\mathbf{0 . 1 1 9}$ & 0.653 & 0.730 & 0.800 & 0.807 \\
$A_{4}$ & 0.166 & 0.030 & $\mathbf{0 . 0 2 7}$ & 1.510 & 0.064 \\
$A_{5}$ & 0.076 & $\mathbf{0 . 0 0 6}$ & 0.008 & 1.409 & 0.171 \\
Results: & $A_{1}\left(\eta_{3}\right)$ & $A_{2}\left(\eta_{5}\right)$ & $A_{3}\left(\eta_{1}\right)$ & $A_{4}\left(\eta_{3}\right)$ & $A_{5}\left(\eta_{2}\right)$ \\
\hline
\end{tabular}

Bold indicates minimum value of each situation with respect to symptoms

Table 5 Values for each considered situation for $\delta_{1}=.5, \delta_{2}=.5$

\begin{tabular}{llllll}
\hline & $\eta_{1}$ & $\eta_{2}$ & $\eta_{3}$ & $\eta_{4}$ & $\eta_{5}$ \\
\hline$A_{1}$ & 0.196 & 0.038 & $\mathbf{0 . 0 0 6}$ & 2.188 & 0.050 \\
$A_{2}$ & 0.051 & 1.360 & 0.675 & 0.685 & $\mathbf{0 . 0 4 9}$ \\
$A_{3}$ & $\mathbf{0 . 1 2 4}$ & 0.660 & 0.737 & 0.789 & 0.809 \\
$A_{4}$ & 0.172 & 0.319 & $\mathbf{0 . 0 2 9}$ & 1.524 & 0.066 \\
$A_{5}$ & 0.079 & $\mathbf{0 . 0 0 6}$ & 0.008 & 1.420 & 1.171 \\
Results: & $A_{1}\left(\eta_{3}\right)$ & $A_{2}\left(\eta_{5}\right)$ & $A_{3}\left(\eta_{1}\right)$ & $A_{4}\left(\eta_{3}\right)$ & $A_{5}\left(\eta_{2}\right)$ \\
\hline
\end{tabular}

Bold indicates minimum value of each situation with respect to symptoms

(2017), Singh (2014) and Nei et al. (2017). Comparative results are appeared in Table 8.

Comparative Analysis: From Table 8, we can easily clear that the proposed picture fuzzy divergence measure gives predictable outcome according to the principal of minimum degree of divergence measure between PFSs, while other divergence measures gave no consistency regardless. They do not ready to perceive the two factors because of the uniformity of divergence measure values. Table 8 gives the results produced by different methods. Two methods are not able to show the clarity about the factor to overcome the situation $A_{1}$ and $A_{5}$. For exampleNei et al. (2017) is not able to suggest for control the situation $A_{4}$, i.e., not able to suggest which factor either clinical management $(\mathrm{CM})$ or monitoring $(\mathrm{MN})$ is suitable to control the situation $A_{4}$. In the same way, Son (2017) is disabled to suggest for handling the situation $A_{1}$, i.e., not able to suggest which preventive factor either clinical management $(\mathrm{CM})$ or country-level coordination (CLC) is suitable to control the situation $A_{1}$.

Further, the proposed measure generates the same results with Wang et al. (2017), which demonstrate that the proposed measure is practical in coronavirus diagnosis. Therefore, our proposed method has strong discrimination and can provide more useful results as contrasted with other divergence measures. 
Table 6 Values for each considered situation for $\delta_{1}=.9, \delta_{2}=.1$

\begin{tabular}{llllll}
\hline & $\eta_{1}$ & $\eta_{2}$ & $\eta_{3}$ & $\eta_{4}$ & $\eta_{5}$ \\
\hline$A_{1}$ & 0.081 & 0.014 & $\mathbf{0 . 0 0 2}$ & 1.775 & 0.019 \\
$A_{2}$ & 0.019 & 1.098 & .554 & 0.521 & $\mathbf{0 . 0 1 8}$ \\
$A_{3}$ & $\mathbf{0 . 0 5 0}$ & 0.511 & 0.578 & 0.586 & 0.630 \\
$A_{4}$ & 0.071 & 0.011 & $\mathbf{0 . 0 1 0}$ & 1.221 & 0.025 \\
$A_{5}$ & 0.030 & $\mathbf{0 . 0 0 2}$ & 0.003 & 1.156 & 0.071 \\
Results: & $A_{1}\left(\eta_{3}\right)$, & $A_{2}\left(\eta_{5}\right)$ & $A_{3}\left(\eta_{1}\right)$ & $A_{4}\left(\eta_{3}\right)$ & $A_{5}\left(\eta_{2}\right)$ \\
\hline
\end{tabular}

Bold indicates minimum value of each situation with respect to symptoms

\subsection{Application to pattern recognition}

For the problems of pattern recognition, it is required for us to identify the pattern which mostly look like the specified pattern by comparing their attributes. The procedure is as follows:

Suppose there are $p$-known patterns $D_{1}, D_{2}, \ldots D_{p}$.

Let the sample be signified by the following PFSs

$D_{p}=\left\{\left(t, \tau_{D_{p}}(t), v_{D_{p}}(t), \pi_{D_{p}}(t), \xi_{D_{p}}(t)\right) / t \in X\right\}$,

where $p=1,2, \ldots, m$ and $j=1,2, \ldots, k$.

An unknown pattern $B$ is

$B=\left\{\left(t, \tau_{B}(t), v_{B}(t), \pi_{B}(t), \xi_{B}(t)\right) / t \in X\right\}$.

Our point is to characterize $B$ is similar to one of the samples $D_{1}, D_{2}, \ldots, D_{p}$. As indicated by the principle of minimum degree of divergence measure between picture fuzzy sets, the way of assigning $B$ to $D_{l}^{*}$ is described by

$l^{*}=\arg \min \left\{\left(D_{p}, B\right)\right\}$.

Example 2 Suppose there be three known patterns $D_{1}, D_{2}$, $D_{3}$ with classifications $C_{1}, C_{2}, C_{3}$ respectively and an unknown pattern $B$ is also given. The patterns are characterized by picture fuzzy sets in $X=\left\{z_{1}, z_{2}, z_{3}, z_{4}, z_{5}\right\}$. Their values are displayed in Table 9. Our objective is to recognize the known pattern which look likes the most with unknown pattern $B$.
Our target is to determine the sample $B$ to one of the patterns $D_{1}, D_{2}, D_{3}$, respectively.

Using the proposed algorithm, one can identify the pattern which closely resembles with the given pattern.

Step 1: The normalized distances for $B$ from the patterns $D_{1}, D_{2}, D_{3}$ are measured by Eq. 31, which are presented in Table 10.

Step 2: $\quad$ The minimum degree of distances between $B$ and $D_{p}, p=1,2,3$ are obtained by Eq. 36 . The minimum distances are darked in Table 10.

Step 3: Using the principle of minimum degree of divergence between PFSs, the known sample which gives the minimum distance with $B$ is determined.

We have calculated the values of $R_{\text {new }}^{\alpha}\left(D_{1}, B\right)$, $R_{\text {new }}^{\alpha}\left(D_{2}, B\right)$ and $R_{\text {new }}^{\alpha}\left(D_{3}, B\right)$ at different values of $\alpha, \delta_{1}, \delta_{2}$. It is preassigned or adjustable number related to individual systems. Let us take an example related to the problem of the environment. Different environmental features such as temperature, pressure and humidity may be denoted as distinct parametric values. Thus, with the existence of parameters, information measure becomes more preferable from application point of view. We may select any value of $\alpha$ within 0 and 1 to achieve the consistent and efficient proposed measure by employing the concept of minimum divergence measure. From Table 10, it is clear that for any parametric value $\alpha, R_{\text {new }}^{\alpha}\left(D_{2}, B\right)$ is the minimum value among $R_{\text {new }}^{\alpha}\left(D_{1}, B\right), R_{\text {new }}^{\alpha}\left(D_{2}, B\right), R_{\text {new }}^{\alpha}\left(D_{3}, B\right)$. Hence, the sample $B$ look likes the same with $D_{2}$, i.e., $B \leftarrow D_{2}$.

Now, we prove the proficiency of the proposed picture fuzzy divergence measure in example 2 by comparing with other existing measures: Hung and Yang (2006), Son and Thong (2017), Ashraf et al. (2019), Wang et al. (2017), Son (2017), Singh (2014) and Nei et al. (2014).

Comparative Analysis: From Table 11, obviously all divergence measures support the sample $D_{2}$ look likes the same with $B$. The results coincide with the proposed measure (3.17). In Fig. 2, blue color presents the distance
Table 7 Outcomes of proposed divergence measure for $\alpha=.2$

\begin{tabular}{lllllll}
\hline Situations & $\delta_{1}=.1$, & $\delta_{1}=.2$, & $\delta_{1}=.3$, & $\delta_{1}=.4$, & $\delta_{1}=.5$, & $\delta_{1}=.9$, \\
& $\delta_{2}=.9$ & $\delta_{2}=.8$ & $\delta_{2}=.7$ & $\delta_{2}=.6$ & $\delta_{2}=.5$ & $\delta_{2}=.1$ \\
\hline$A_{1}$ & CLC & CLC & CLC & CLC & CLC & CLC \\
$A_{2}$ & IPP & IPP & IPP & IPP & IPP & IPP \\
$A_{3}$ & CM & CM & CM & CM & CM & CM \\
$A_{4}$ & CLC & CLC & CLC & CLC & CLC & CLC \\
$A_{5}$ & MN & MN & MN & MN & MN & MN \\
\hline
\end{tabular}


Table 8 Comparison of proposed and existed picture fuzzy divergence measures
Table 9 Known and unknown pattern values

\begin{tabular}{llllll}
\hline Divergence measures & $A_{1}$ & $A_{2}$ & $A_{3}$ & $A_{4}$ & $A_{5}$ \\
\hline Proposed measure & CLC & IPP & CM & CLC & MN \\
Hung and Yang (2006) & CM & CE & CLC & CM & CM \\
Son and Thong (2017) & CM & CE & CLC & CM & CM \\
Ashraf et al. (2019) & MN & CE & CLC & CM & CM \\
Wang et al. (2017) & CLC & IPP & CM & CLC & MN \\
Son (2017) & eithor MN or CLC & CE & MN & CM & MN \\
Singh (2014) & MN & CE & CLC & CM & CM \\
Nei et al. (2017) & MN & CE & CLC & eithor CM or MN & MN \\
\hline
\end{tabular}

\begin{tabular}{llllll}
\hline Samples & $z_{1}$ & $z_{2}$ & $z_{3}$ & $z_{4}$ & $z_{5}$ \\
& $\left(\tau_{z_{1}}, v_{z_{1}}, \pi_{z_{1}}, \xi_{z_{1}}\right)$ & $\left(\tau_{z_{2}}, v_{z_{2}}, \pi_{z_{2}}, \xi_{z_{2}}\right)$ & $\left(\tau_{z_{3}}, v_{z_{3}}, \pi_{z_{3}}, \xi_{z_{3}}\right)$ & $\left(\tau_{z_{4}}, v_{z_{4}}, \pi_{z_{4}}, \xi_{z_{4}}\right)$ & $\left(\tau_{z_{5}}, v_{z_{5}}, \pi_{z_{5}}, \xi_{z_{5}}\right)$ \\
\hline$B$ & $(0.2,0.6,0.1,0.1)$ & $(0.3,0.1,0.4,0.2)$ & $(0.2,0.2,0.5,0.1)$ & $(0.2,0.7,0.0,0.1)$ & $(0.2,0.5,0.1,0.2)$ \\
$D_{1}$ & $(0.3,0.2,0.1,0.4)$ & $(0.2,0.5,0.3,0.0)$ & $(0.1,0.3,0.3,0.3)$ & $(0.1,0.2,0.7,0.0)$ & $(0.1,0.6,0.2,0.1)$ \\
$D_{2}$ & $(0.2,0.1,0.6,0.1)$ & $(0.1,0.4,0.4,0.1)$ & $(0.3,0.5,0.1,0.1)$ & $(0.0,0.3,0.5,0.2)$ & $(0.3,0.3,0.2,0.2)$ \\
$D_{3}$ & $(0.4,0.1,0.3,0.2)$ & $(0.2,0.3,0.4,0.1)$ & $(0.2,0.5,0.1,0.2)$ & $(0.1,0.5,0.2,0.2)$ & $(0.4,0.5,0.1,0.0)$ \\
\hline
\end{tabular}

between $D_{1}$ and $B$, red color presents the distance between $D_{2}$ and $B$ and green color presents the distance between $D_{3}$ and $B$. Also, Fig. 2 reveals that all existed picture fuzzy divergence measures support the sample $D_{2}$ according to a minimum degree of divergence between two picture fuzzy sets. Therefore, with the presence of parameter proposed measure becomes more compatible and broaden its extent of implementation as compare with other methods. This reveals that execution of proposed measure is considerably superior.

\subsection{Advantages of the proposed measure}

The following advantages have been taken into account from the proposed pictur fuzzy divergence measure:

- As mentioned above, the PFS is one of the generalization of the classic set, fuzzy set, intuitionistic fuzzy set. Numerous circumstances occur which are not properly controlled in IFS. To overcome this situation, Picture fuzzy set theory is one of the more broad and can handle indeterminate information, which exists commonly in real-life situations. Hence, Picture fuzzy divergence measure is more suitable in real scientific applications.

- Various interpretations to $\alpha, \delta_{1}, \delta_{2}$ is given for consistency and efficiency of the proposed measure. The presence of the parameters in the proposed measure makes it progressively reliable and widens its extent of applications as contrasted with other measures. This
Table 10 Outcomes of proposed picture fuzzy divergence measure

\begin{tabular}{llll}
\hline$R_{\text {new }}^{\alpha}\left(D_{p}, B\right) ; p=1,2,3$ & $R_{\text {new }}^{\alpha}\left(D_{1}, B\right)$ & $R_{\text {new }}^{\alpha}\left(D_{2}, B\right)$ & $R_{\text {new }}^{\alpha}\left(D_{3}, B\right)$ \\
\hline$\delta_{1}=.1, \delta_{2}=.9$ & 0.593 & $\mathbf{0 . 0 2 4}$ & 0.075 \\
$\delta_{1}=.2, \delta_{2}=.8$ & 0.684 & $\mathbf{0 . 0 4 1}$ & 0.126 \\
$\delta_{1}=.3, \delta_{2}=.7$ & 0.734 & $\mathbf{0 . 0 5 3}$ & 0.154 \\
$\delta_{1}=.4, \delta_{2}=.6$ & 1.021 & $\mathbf{0 . 0 6 0}$ & 0.178 \\
$\delta_{1}=.5, \delta_{2}=.5$ & 0.769 & $\mathbf{0 . 0 6 2}$ & 0.184 \\
$\delta_{1}=.6, \delta_{2}=.4$ & 1.021 & $\mathbf{0 . 0 6 0}$ & 0.178 \\
$\delta_{1}=.7, \delta_{2}=.3$ & 0.734 & $\mathbf{0 . 0 5 3}$ & 0.154 \\
$\delta_{1}=.8, \delta_{2}=.2$ & 0.684 & $\mathbf{0 . 0 4 1}$ & 0.126 \\
$\delta_{1}=.9, \delta_{2}=.1$ & 0.593 & $\mathbf{0 . 0 2 4}$ & 0.075 \\
\hline Bold
\end{tabular}

Bold indicates minimum value of each situation with respect to symptoms

shows that the performance of the proposed picture fuzzy divergence measure is significantly flexible.

- The proposed picture fuzzy divergence measure is more generalized and appropriate to solve financial, medical and other multicriteria decision-making problems.

\section{Conclusions}

In this article, we have developed a new picture fuzzy divergence measure based on Jensen-Tsallis information measure. The idea has been extended of Tsallis entropy and Jensen inequality to present a new picture fuzzy divergence 
Table 11 Calculated values of proposed and existed picture fuzzy divergence measures

\begin{tabular}{lllll}
\hline Divergence measures & $\left(D_{1}, B\right)$ & $\left(D_{2}, B\right)$ & $\left(D_{3}, B\right)$ & Classification Results \\
\hline Proposed measure & 0.593 & $\mathbf{0 . 0 2 4}$ & 0.075 & $D_{2}$ \\
Hung and Yang (2006) & 0.452 & $\mathbf{0 . 4 0 1}$ & 0.416 & $D_{2}$ \\
Son and Thong (2017) & 0.784 & $\mathbf{0 . 7 3 0}$ & 0.882 & $D_{2}$ \\
Ashraf et al. (2019) & 0.224 & $\mathbf{0 . 0 5 7}$ & 0.147 & $D_{2}$ \\
Wang et al. (2017) & 0.352 & $\mathbf{0 . 1 0 1}$ & 0.116 & $D_{2}$ \\
Son (2017) & 0.586 & $\mathbf{0 . 0 2 3}$ & 0.223 & $D_{2}$ \\
Singh (2014) & 0.083 & $\mathbf{0 . 0 4 3}$ & 0.080 & $D_{2}$ \\
Nei et al. (2017) & 0.650 & $\mathbf{0 . 6 0 0}$ & 0.650 & $D_{2}$ \\
\hline
\end{tabular}

Bold indicates minimum value of each situation with respect to symptoms

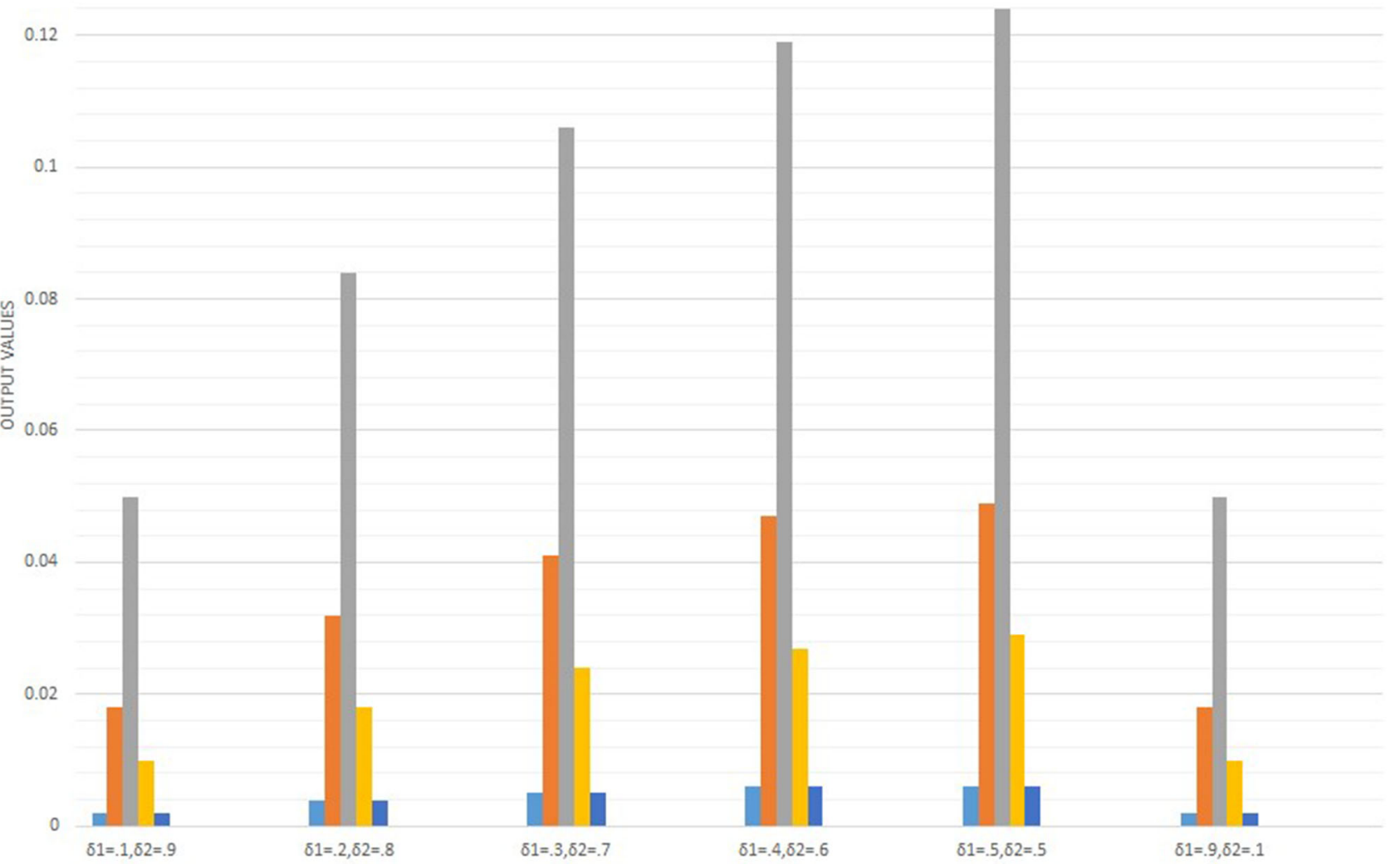

Fig. 1 Outcomes of proposed picture fuzzy divergence measure

measure. Furthermore, a few key properties and particular cases are also studied. The outcome of the proposed measure is compared to other existing methods in literature. To build up the sufficiency, flexibility of proposed measure, some counter examples are solved in the context of COVID-19 and pattern recognition. It is summarized that the developed method can provide extraordinary evaluation results because of the presence of the parameter. The main advantages of the proposed method are the computation simplicity for picture fuzzy sets.

To discuss the future prospects of the proposed research, it can be further extended to more general sets such as interval-valued PFSs, and complex-valued fuzzy sets. Apart from this, we can also apply the applications of 


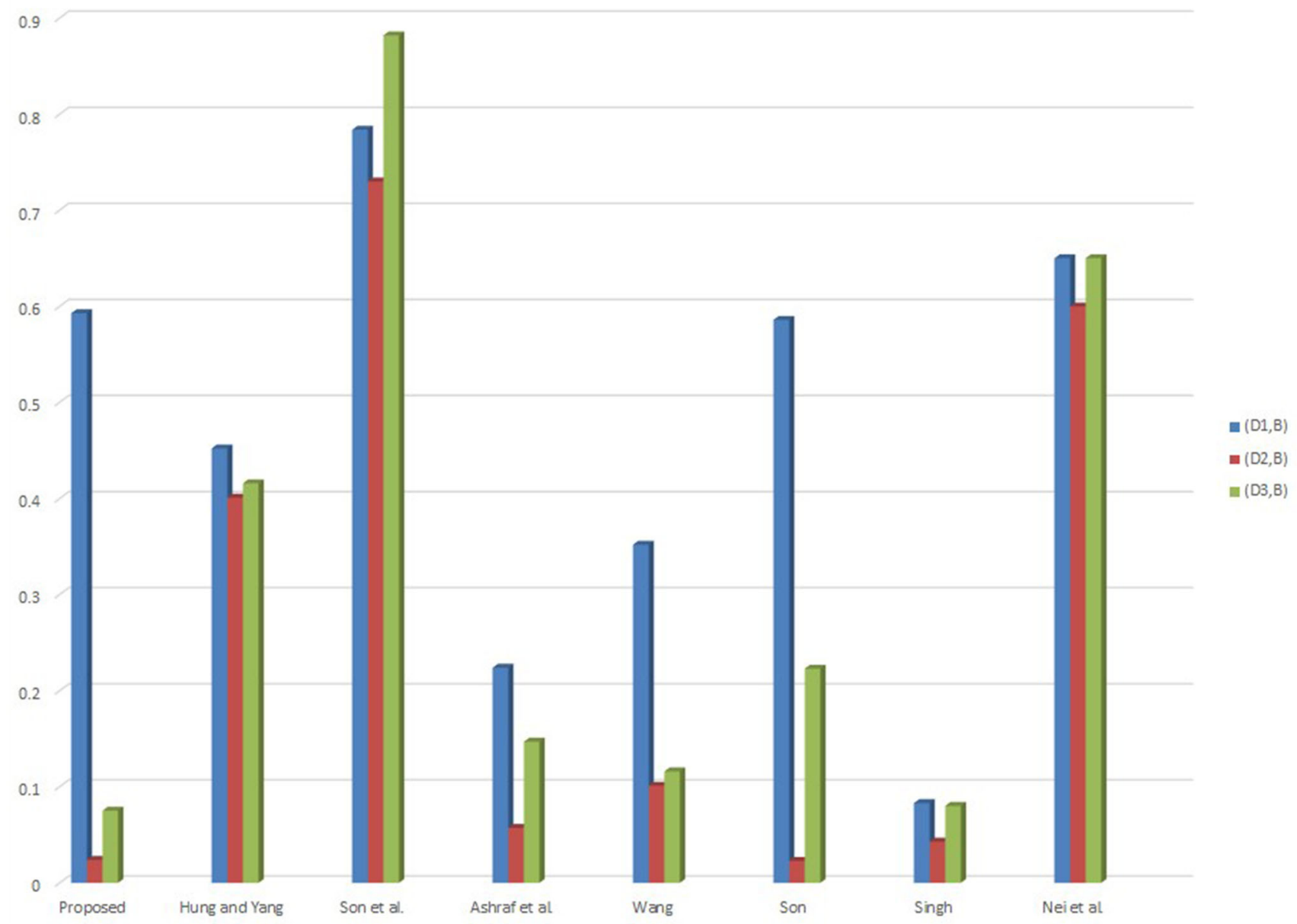

Fig. 2 Sensitive analysis of proposed and existed picture fuzzy divergence measures

proposed measure in other MCDM problems such as remote sensing, speech recognition, risk analysis, weather forecasting and so on.

Acknowledgements The authors are thankful to the editor and the anonymous reviewers for their precious suggestions and comments which improved this manuscript and enhanced our knowledge.

\section{Compliance with ethical standards}

Conflict of Interest The authors declare that they have no conflict of interest.

\section{References}

Atanassov KT (1986) Intuitionistic fuzzy sets. Fuzzy Sets Syst 20:87-96

Atanassov KT (1999) Intuitionistic fuzzy sets. Physica, Heidelberg, Germany
Ashraf S, Mahmood T, Abdullah S, Khan Q (2019) Different approaches to multi-criteria group decision making problems for picture fuzzy environment. Bull Braz Math Soc 50(2):373-397

Ashraf S, Abdullah S, Mahmood T, Aslam M (2019) Cleaner production evaluation in gold mines using novel distance measure method with cubic picture fuzzy numbers. Int J Fuzzy Syst 21(8):2448-2461

Burbea J, Rao CR (1982) On the convexity of some divergence measures based on entropy function. IEEE Trans Inf Theo 28:489-495

Chen SM, Chen SW (2014) Fuzzy forecasting based on two-factors second-order fuzzy-trend logical relationship groups and the probabilities of trends of fuzzy logical relationships. IEEE Trans Cybern 45(3):391-403

Chen SM, Chang CH (2016) Fuzzy multiattribute decision making based on transformation techniques of intuitionistic fuzzy values and intuitionistic geometric averaging operators. Inf Sci 352:133-149

Chen SM, Cheng SH, Lan TC (2016) Multicriteria decision making based on the TOPSIS method and similarity measures between intuitionistic fuzzy values. Inf Sci 367:279-295

Chen SM, Chu HP, Sheu TW (2012) TAIEX forecasting using fuzzy time series and automatically generated weights of multiple 
factors. IEEE Trans Syst Man Cybern Part A Syst Hum 42(6):1485-1495

Cuong BC, Kreinovich V (2014) Picture fuzzy sets. J Comput Sci Cybern 30(4):409-416

Hung WL, Yang MS (2008) On the j-divergence of intuitionistic fuzzy sets and its application to pattern recognition. Inf Sci 178(6):1641-1650

Hung WL, Yang MS (2006) Fuzzy entropy on intuitionistic fuzzy sets. Int J Intell Syst 21:443-451

Jiang YC, Tang Y, Wang J, Tang S (2009) Reasoning with intuitionistic fuzzy rough description logics. Inf Sci 179:2362-2378

Joshi R, Kumar S (2018) A novel fuzzy decision making method using entropy weights based correlation coefficients under intuitionistic fuzzy environment. Int J Fuzzy Syst. https://doi. org/10.1007/s40815-018-0538-8

Joshi R, Kumar S (2019) Jensen-Tsalli's intuitionistic fuzzy divergence measure and its applications in medical analysis and pattern recognition. Int J Uncertainty Fuzziness Knowl Based Syst 27(1):145-169

Joshi R (2020) A novel decision-making method using R-norm concept and VIKOR approach under picture fuzzy environment. Expert Syst Appl 147:113228

Kadian R, Kumar S (2020) Renyi's-Tsallis fuzzy divergence measure and its applications to pattern recognition and fault detection. J Int Fuzzy Syst 39(1):731-752

Kadian R, Kumar S (2020) Jensen-Renyi's-Tsallis fuzzy divergence information measure with its applications. Comm Math Stat. https://doi.org/10.1007/s40304-020-00228-1

Kadian R, Kumar S (2020) A novel intuitionistic Renyi's-Tsallis discriminant information measure and its applications in decision making. Granul Comput. https://doi.org/10.1007/s41066020-00237-z

Kullback S, Leibler RA (1951) On information and sufficiency. Annals Math Stat. 22(1):79-86

Li X, Chen X (2018) D-intuitionistic hesitant fuzzy sets and their application in multi attribute decision making. Cognit Comput 10(3):496-505

Lin J (1991) Divergence measure based on Shannon entropy. IEEE Trans Inf Theo 37(1):145-151

Liang R, He S, Wang J, Chen K (2019) An extended MABAC method for multi-criteria group decision-making problems based on correlation inputs of intuitionistic fuzzy information. Comput Appl Math 38(3):112 https://https://doi.org/10.1007/s40314-0190886-5

Nie RX, Wang JQ, Li L (2017) A shareholder voting method for proxy advisory firm selection based on 2-tuple linguistic picture preference relation. Appl Soft Comput 60:520-539

Phong PH, Cuong BC (2017) Multi-criteria decision making with picture linguistic numbers. VNU J Sci Comput SCi Commun Eng 32:39-53

Qiyas M, Abdullah S, Ashraf S, Aslam M (2020) Utilizing linguistic picture fuzzy aggregation operators for multiple-attribute decision-making problems. Int J Fuzzy Syst 22(1):310-320

Szmidt E, Kacprzyk J (2000) Distance between intuitionistic fuzzy sets. Fuzzy Sets Syst 114:505-518
Son LH, Phong PH (2016) On the performance evaluation of intuitionistic vector similarity measures for medical diagnosis. J Intell Fuzzy Syst 31:1597-1608

Son LH, Thong PH (2017) Some novel hybrid forecast methods based on picture fuzzy clustering for weather now casting from satellite image sequences. Appl Intel 46:1-15

Son LH, Viet PV (2017) Picture inference system: a new inference system on picture fuzzy set. Appl Intell 46:652-669

Son LH (2016) Generalized picture distance measure and applications to picture fuzzy clustering. Appl Soft Comput 46:284-295

Sohail A, Nutini A (2020) Forecasting the timeframe of coronavirus and human cells interaction with reverse engineering. Prog Biophys Mol Bio 155:29-35

Shannon CE (1948) The mathematical theory of communication. Bell Syst Tech J 27(379-423):623-656

Singh P (2014) Correlation coefficients for picture fuzzy sets. J Intell Fuzzy Syst 27:2857-2868

Toğaçar M, Ergen B, Cömert Z (2020) COVID-19 detection using deep learning models to exploit social mimic optimization and structured chest X-ray images using fuzzy color and stacking approaches. Comput Biol Med 122:103805

Tuite AR, Fisman DN, Greer AL (2020) Mathematical modelling of COVID-19 transmission and mitigation strategies in the population of Ontario. Canada. Can Med Assoc J 192(19):E497-E505

Tsallis C (1988) Possible generalization of Boltzman-Gibbs statistics. J Stat Phys 52:480-487

Vlachos IK, Sergiadis GD (2007) Intuitionistic fuzzy information applications to the pattern recognition. Pattern Recogn Lett 28(2):197-206

Wei GW (2018) Some more similarity measures for picture fuzzy sets and their applications. Iranian J fuzzy syst 15(1):77-89

Wang C, Zhou X, Tu H, Tao S (2017) Some geometric aggregation operators based on picture fuzzy sets and their application in multiple attribute decision making. Ital $\mathrm{J}$ pure Appl Math 37:477-492

Wei G (2018) Picture fuzzy aggregation operators and their application to multiple attribute decision making. Fund Inf 37:271-320

Xu ZS, Xia MM (2010) Some new similarity measures for intuitionistic fuzzy values and their application in group decision making. J Syst Sci Eng 19:430-452

Zadeh LA (1965) Fuzzy sets. Inf. Control 8:338-353

Zadeh LA (1975) The concept of linguistic variable and its application to approximate reasoning-1. Inf Sci 8:199-249

Zeng S, Chen SM, Kuo LW (2019) Multiattribute decision making based on the novel score function of intuitionistic fuzzy values and modified VIKOR method. Inf Sci 488:76-92

Zeng W, Yu F, Yu X, Chen H, Wu S (2009) Entropy on intuitionistic fuzzy set based on similarity measure. Int J Innovat Comput Inf Cont 5(12):4737-4744

Publisher's Note Springer Nature remains neutral with regard to jurisdictional claims in published maps and institutional affiliations. 